\title{
Studies of transverse momentum dependent parton distributions and Bessel weighting
}

M. Aghasyan, ${ }^{a, b}$ H. Avakian, ${ }^{c}$ E. De Sanctis, ${ }^{a}$ L. Gamberg, ${ }^{d}$ M. Mirazita, ${ }^{a}$ B. Musch, ${ }^{e}$ A. Prokudin ${ }^{c}$ and P. Rossi ${ }^{a, c}$

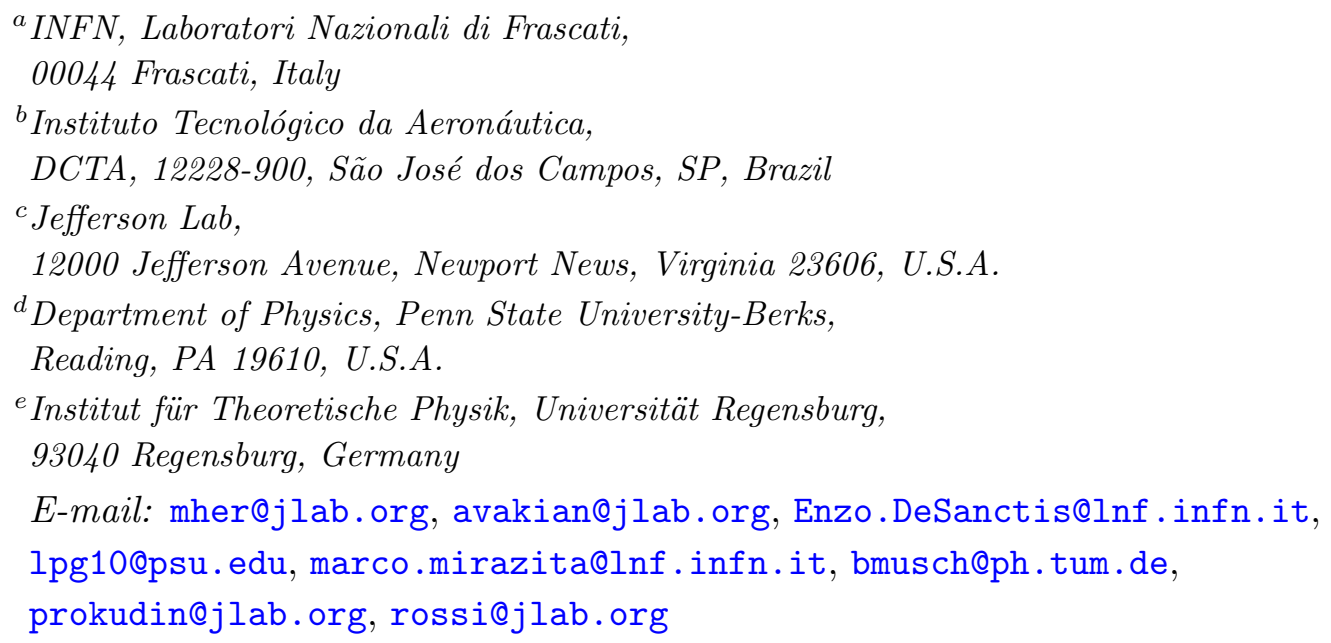

ABSTRACT: In this paper we present a new technique for analysis of transverse momentum dependent parton distribution functions, based on the Bessel weighting formalism. The procedure is applied to studies of the double longitudinal spin asymmetry in semiinclusive deep inelastic scattering using a new dedicated Monte Carlo generator which includes quark intrinsic transverse momentum within the generalized parton model. Using a fully differential cross section for the process, the effect of four momentum conservation is analyzed using various input models for transverse momentum distributions and fragmentation functions. We observe a few percent systematic offset of the Bessel-weighted asymmetry obtained from Monte Carlo extraction compared to input model calculations, which is due to the limitations imposed by the energy and momentum conservation at the given energy $/ Q^{2}$. We find that the Bessel weighting technique provides a powerful and reliable tool to study the Fourier transform of TMDs with controlled systematics due to experimental acceptances and resolutions with different TMD model inputs.

KeYwords: Deep Inelastic Scattering, Parton Model, QCD

ARXIV EPRINT: 1409.0487 


\section{Contents}

1 Introduction 1

2 Extraction of TMDs using Bessel weighting 3

2.1 The cross section for semi-inclusive deep inelastic scattering 3

2.2 Bessel weighting of experimental observables 5

3 Fully differential Monte Carlo for SIDIS $\quad 7$

3.1 The Monte Carlo and the generalized parton model $\quad 7$

$\begin{array}{ll}3.2 \text { Kinematical distributions } & 11\end{array}$

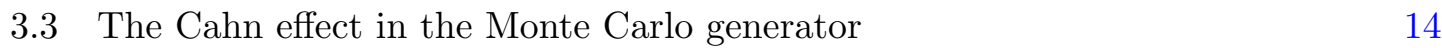

4 Bessel weighted double spin asymmetry $\quad 14$

$\begin{array}{lll}4.1 & \text { Results from the Monte Carlo } & 14\end{array}$

$\begin{array}{lll}4.2 & \text { Interpretation of the results } & 18\end{array}$

$\begin{array}{llr}5 & \text { Conclusions } & 19\end{array}$

$\begin{array}{lr}\text { A Bessel weighting } & 20\end{array}$

B Error calculations $\quad 22$

C Bootstrap technique for weighted Poisson events 23

\section{Introduction}

The study of the spin structure of protons and neutrons is one of the central issues in hadron physics, with many dedicated experiments, recent (HERMES at DESY, CLAS and HallA at JLAB), running (COMPASS at CERN, STAR and PHENIX at RHIC), approved (JLab $12 \mathrm{GeV}$ upgrade [1], COMPASS-II [2]) or planned (Electron Ion Collider [3-5]). The Transverse Momentum Dependent (TMD) parton distribution functions and fragmentation functions play a crucial role in gathering and interpreting information of a true "3-dimensional" imaging of the nucleon. These Transverse Momentum Dependent distribution and fragmentation functions (collectively here called "TMDs") can be accessed in several types of processes, one of the most important is single particle hadron production in Semi-Inclusive Deep Inelastic Scattering (SIDIS) of leptons on nucleons. A significant amount of data on spin-azimuthal distributions of hadrons in SIDIS, providing access to TMDs has been accumulated in recent years by several collaborations, including HERMES, COMPASS and Halls A,B and C at JLab [6-15]. At least an order of magnitude more data is expected in coming years of running of JLab 12 [1]. 
A rigorous basis for studies of TMDs in SIDIS is provided by TMD factorization in QCD, which has been established in refs. [16-23] for leading twist single hadron production with transverse momentum of the produced hadron being much smaller than the hard scattering scale, and the order of $\Lambda_{Q C D}$, that is $\Lambda_{Q C D}^{2}<P_{h \perp}^{2} \ll Q^{2}$. In this kinematic domain the SIDIS cross section can be expressed in terms of structure functions encoding the strong-interaction dynamics of the hadronic sub-process $\gamma^{*}+p \rightarrow h+X$ [24-27], which are given by convolutions of a hard scattering cross section and TMDs. However the extraction of TMDs as a function of the light-cone fraction $x$ and transverse momentum $k_{\perp}$ from single and double spin azimuthal asymmetries is hindered by the fact that observables are complicated convolutions in momentum space making the flavor decomposition of the underlying TMDs a model dependent procedure.

Based on TMD factorization theorems, experimentally measured cross sections are expressed as convolutions of TMDs where $k_{\perp}$ dependence is integrated over and related to the measured value of $P_{h \perp}$. A reliable method to directly access the $k_{\perp}$ dependence of TMDs is very desirable. However, various assumptions involved in modern extractions of TMDs from available data rely on conjectures of the transverse momentum dependence of distribution and fragmentation functions [28-38] making estimates of systematic errors due to those assumptions extremely challenging.

In a paper by Boer, Gamberg, Musch, and Prokudin [39], a new technique has been proposed called Bessel weighting, which relies on a model-independent deconvolution of structure functions in terms of Fourier transforms of TMDs from observed azimuthal moments in SIDIS with polarized and unpolarized targets. In this paper, we apply the Bessel weighting procedure to present an extraction of Fourier transforms of TMDs from a Monte Carlo event generator. As an application of this procedure we consider the ratio of helicity $g_{1 L}$, and unpolarized $f_{1}$ TMDs from the double longitudinally polarization asymmetry.

This paper is organized as follows: we begin our discussion in section 2 with a brief review of the formalism of the SIDIS cross section and its representation in both momentum and Fourier conjugate $b_{T}$ space. The latter representation lends itself to a discussion of the Bessel weighting formalism [39]. We review its merits in studying the transverse structure of the nucleon and present a description of the experimental procedure to study TMDs using Bessel weighting which provides a new tool to study nucleon structure. In section 3 we introduce a fully differential Monte Carlo generator which has been developed to test the procedure for extraction of TMDs from SIDIS. As a test of the quality of our constructed Monte Carlo, in section 3.3 we present a study of the Cahn effect $[40,41]$ contribution to the average $\langle\cos \phi\rangle$ moment in SIDIS. In section 4 we present the extraction of the double spin asymmetry $A_{L L}\left(b_{T}\right)$, defined as the ratio of the difference and the sum of electro-production cross sections for anti-parallel and parallel configurations of lepton and nucleon spins using the Bessel weighting procedure. The effects of different model inputs and experimental resolutions and acceptances on extracted TMDs are investigated. Finally in section 5 we draw some conclusions of the present analysis and outline steps for future work. 


\section{Extraction of TMDs using Bessel weighting}

\subsection{The cross section for semi-inclusive deep inelastic scattering}

The SIDIS cross section can be expressed in a model independent way in terms of a set of 18 structure functions [24, 25, 27, 42-44],

$$
\begin{aligned}
& \frac{d \sigma}{d x d y d \psi d z d \phi_{h} d\left|\boldsymbol{P}_{h \perp}\right|^{2}}=\frac{\alpha^{2}}{x y Q^{2}} \frac{y^{2}}{2(1-\varepsilon)}\left(1+\frac{\gamma^{2}}{2 x}\right)\left\{F_{U U, T}+\varepsilon F_{U U, L}\right. \\
& +\quad \sqrt{2 \varepsilon(1+\varepsilon)} \cos \phi_{h} F_{U U}^{\cos \phi_{h}}+\varepsilon \cos \left(2 \phi_{h}\right) F_{U U}^{\cos 2 \phi_{h}} \\
& +\lambda_{e} \sqrt{2 \varepsilon(1-\varepsilon)} \sin \phi_{h} F_{L U}^{\sin \phi_{h}} \\
& +S_{\|}\left[\sqrt{2 \varepsilon(1+\varepsilon)} \sin \phi_{h} F_{U L}^{\sin \phi_{h}}+\varepsilon \sin \left(2 \phi_{h}\right) F_{U L}^{\sin 2 \phi_{h}}\right] \\
& +S_{\|} \lambda_{e}\left[\sqrt{1-\varepsilon^{2}} F_{L L}+\sqrt{2 \varepsilon(1-\varepsilon)} \cos \phi_{h} F_{L L}^{\cos \phi_{h}}\right] \\
& +\left|\boldsymbol{S}_{\perp}\right|\left[\sin \left(\phi_{h}-\phi_{S}\right)\left(F_{U T, T}^{\sin \left(\phi_{h}-\phi_{S}\right)}+\varepsilon F_{U T, L}^{\sin \left(\phi_{h}-\phi_{S}\right)}\right)\right. \\
& +\varepsilon \sin \left(\phi_{h}+\phi_{S}\right) F_{U T}^{\sin \left(\phi_{h}+\phi_{S}\right)}+\varepsilon \sin \left(3 \phi_{h}-\phi_{S}\right) F_{U T}^{\sin \left(3 \phi_{h}-\phi_{S}\right)} \\
& \left.+\sqrt{2 \varepsilon(1+\varepsilon)} \sin \phi_{S} F_{U T}^{\sin \phi_{S}}+\sqrt{2 \varepsilon(1+\varepsilon)} \sin \left(2 \phi_{h}-\phi_{S}\right) F_{U T}^{\sin \left(2 \phi_{h}-\phi_{S}\right)}\right] \\
& +\left|\boldsymbol{S}_{\perp}\right| \lambda_{e}\left[\sqrt{1-\varepsilon^{2}} \cos \left(\phi_{h}-\phi_{S}\right) F_{L T}^{\cos \left(\phi_{h}-\phi_{S}\right)}+\sqrt{2 \varepsilon(1-\varepsilon)} \cos \phi_{S} F_{L T}^{\cos \phi_{S}}\right. \\
& \left.\left.+\sqrt{2 \varepsilon(1-\varepsilon)} \cos \left(2 \phi_{h}-\phi_{S}\right) F_{L T}^{\cos \left(2 \phi_{h}-\phi_{S}\right)}\right]\right\}
\end{aligned}
$$

where the first two subscripts of the structure functions $F_{X Y}$ indicate the polarization of the beam and target, and in certain cases, a third sub-script in $F_{X Y, Z}$ indicates the polarization of the virtual photon. The structure functions depend on the the scaling variables $x, z$, the four momentum $Q^{2}=-q^{2}$, where $q=l-l^{\prime}$ is the momentum of the virtual photon, and $l$ and $l^{\prime}$ are the 4-momenta of the incoming and outgoing leptons, respectively. $P_{h \perp}$ is the transverse momentum component of the produced hadron with respect to the virtual photon direction.

The scaling variables have the standard definitions, $x=Q^{2} / 2(P \cdot q), y=(P \cdot q) /(P \cdot l)$, and $z=\left(P \cdot P_{h}\right) /(P \cdot q)$. Further, in eq. (2.1) $\alpha$ is the fine structure constant; the angle $\psi$ is the azimuthal angle of $\ell^{\prime}$ around the lepton beam axis with respect to an arbitrary fixed direction [44], and $\phi_{h}$ is the azimuthal angle between the scattering plane formed by the initial and final momenta of the electron and the production plane formed by the transverse momentum of the observed hadron and the virtual photon, whereas $\phi_{S}$ is the azimuthal angle of the transverse spin in the scattering plane [45]. Finally, $\varepsilon$ is the ratio of longitudinal and transverse photon fluxes [27].

At tree-level, in a parton model factorization framework [25, 27, 43], the various structure functions in the cross section are written as convolutions of the TMDs which relate transverse momenta of the active partons and produced hadron. For our purposes, the 
unpolarized and double longitudinal polarized structure functions are

$$
\begin{aligned}
F_{U U, T} & =x \sum_{a} e_{a}^{2} \int d^{2} \boldsymbol{p}_{\perp} d^{2} \boldsymbol{k}_{\perp} \delta^{(2)}\left(z \boldsymbol{k}_{\perp}+\boldsymbol{p}_{\perp}-\boldsymbol{P}_{h \perp}\right) f_{1}^{a}\left(x, \boldsymbol{k}_{\perp}^{2}\right) D_{1}^{a}\left(z, \boldsymbol{p}_{\perp}^{2}\right) \\
F_{L L} & =x \sum_{a} e_{a}^{2} \int d^{2} \boldsymbol{p}_{\perp} d^{2} \boldsymbol{k}_{\perp} \delta^{(2)}\left(z \boldsymbol{k}_{\perp}+\boldsymbol{p}_{\perp}-\boldsymbol{P}_{h \perp}\right) g_{1 L}^{a}\left(x, \boldsymbol{k}_{\perp}^{2}\right) D_{1}^{a}\left(z, \boldsymbol{p}_{\perp}^{2}\right)
\end{aligned}
$$

where $\boldsymbol{k}_{\perp}$ is the intrinsic transverse momentum of the struck quark, and $\boldsymbol{p}_{\perp}$ is the transverse momentum of the final state hadron relative to the fragmenting quark $k^{\prime}$ (see figure 1 ). $f_{1}^{a}\left(x, \boldsymbol{k}_{\perp}^{2}\right), g_{1 L}^{2}$ and $D^{a}\left(z, \boldsymbol{p}_{\perp}^{2}\right)$ represent TMD PDFs and fragmentation functions respectively of flavor $a, e_{a}$ is the fractional charge of the struck quark or anti-quark and the summation runs over quarks and anti-quark flavors $a$.

Measurements of the transverse momentum $P_{h \perp}$ of final state hadrons in SIDIS with polarized leptons and nucleons provide access to transverse momentum dependence of TMDs. Recent measurements of multiplicities and double spin asymmetries as a function of the final transverse momentum of pions in SIDIS at COMPASS [46], HERMES [47], and JLab [13-15] suggest that transverse momentum distributions depend on the polarization of quarks and possibly also on their flavor [38] (see also discussion in ref. [48]). Calculations of transverse momentum dependence of TMDs in different models [49-52] and on the lattice $[53,54]$ also indicate that the dependence of transverse momentum distributions on the quark polarization and flavor maybe significant. Larger intrinsic transverse momenta of sea-quarks compared to valence quarks have been discussed in an effective model of the low energy dynamics resulting from chiral symmetry breaking in QCD [55].

As stated above, the various assumptions on transverse momentum dependence of distributions on spin and flavor of quarks however make phenomenological fits very challenging. To minimize these model assumptions, Kotzinian and Mulders [56] suggested using so called $P_{h \perp}$-weighted asymmetries, where the unknown $k_{\perp}$-dependencies of TMDs are integrated out, thus providing access to moments of TMDs. However, the $P_{h \perp}$-weighted asymmetries introduce a significant challenge to both theory and experiment. For example, the weighting with $P_{h \perp}$ emphasizes the kinematical region with higher $P_{h \perp}$, where the statistics are poor and systematics from detector acceptances are difficult to control and at the same time theoretical description in terms of TMDs breaks down.

The method of Bessel weighting [39] addresses these experimental and theoretical issues. First, Bessel weighted asymmetries are given in terms of simple products of Fourier transformed TMDs without imposing any model assumptions of the their transverse momentum dependence. Secondly, Bessel weighting regularizes the ultraviolet divergences resulting from unbound momentum integration that arises from conventional weighting. Further, in this paper we will demonstrate that they provide a new experimental tool to study the TMD content to the SIDIS cross section that minimize the transverse momentum model dependencies inherent in conventional extractions of TMDs. Also they suppress the kinematical regions where cross sections are small and statistics are poor [39]. 
We begin the discussion of Bessel weighting by re-expressing the SIDIS cross section as a Bessel weighted integral in $b_{T}$ space [39]:

$$
\begin{aligned}
& \frac{d \sigma}{d x d y d \psi d z_{h} d \phi_{h} d\left|\boldsymbol{P}_{h \perp}\right|^{2}}= \\
& \frac{\alpha^{2}}{x y Q^{2}} \frac{y^{2}}{(1-\varepsilon)}\left(1+\frac{\gamma^{2}}{2 x}\right) \int \frac{d\left|\boldsymbol{b}_{T}\right|}{(2 \pi)}\left|\boldsymbol{b}_{T}\right|\left\{J_{0}\left(\left|\boldsymbol{b}_{T}\right|\left|\boldsymbol{P}_{h \perp}\right|\right) \mathcal{F}_{U U, T}+\varepsilon J_{0}\left(\left|\boldsymbol{b}_{T}\right|\left|\boldsymbol{P}_{h \perp}\right|\right) \mathcal{F}_{U U, L}\right. \\
& +\sqrt{2 \varepsilon(1+\varepsilon)} \cos \phi_{h} J_{1}\left(\left|\boldsymbol{b}_{T}\right|\left|\boldsymbol{P}_{h \perp}\right|\right) \mathcal{F}_{U U}^{\cos \phi_{h}}+\varepsilon \cos \left(2 \phi_{h}\right) J_{2}\left(\left|\boldsymbol{b}_{T}\right|\left|\boldsymbol{P}_{h \perp}\right|\right) \mathcal{F}_{U U}^{\cos \left(2 \phi_{h}\right)} \\
& +\lambda_{e} \sqrt{2 \varepsilon(1-\varepsilon)} \sin \phi_{h} J_{1}\left(\left|\boldsymbol{b}_{T} \| \boldsymbol{P}_{h \perp}\right|\right) \mathcal{F}_{L U}^{\sin \phi_{h}} \\
& +\quad S_{\|}\left[\sqrt{2 \varepsilon(1+\varepsilon)} \sin \phi_{h} J_{1}\left(\left|\boldsymbol{b}_{T} \| \boldsymbol{P}_{h \perp}\right|\right) \mathcal{F}_{U L}^{\sin \phi_{h}}+\varepsilon \sin \left(2 \phi_{h}\right) J_{2}\left(\left|\boldsymbol{b}_{T}\right|\left|\boldsymbol{P}_{h \perp}\right|\right) \mathcal{F}_{U L}^{\sin 2 \phi_{h}}\right] \\
& +S_{\|} \lambda_{e}\left[\sqrt{1-\varepsilon^{2}} J_{0}\left(\left|\boldsymbol{b}_{T}\right|\left|\boldsymbol{P}_{h \perp}\right|\right) \mathcal{F}_{L L}+\sqrt{2 \varepsilon(1-\varepsilon)} \cos \phi_{h} J_{1}\left(\left|\boldsymbol{b}_{T}\right|\left|\boldsymbol{P}_{h \perp}\right|\right) \mathcal{F}_{L L}^{\cos \phi_{h}}\right] \\
& +\left|\boldsymbol{S}_{\perp}\right|\left[\sin \left(\phi_{h}-\phi_{S}\right) J_{1}\left(\left|\boldsymbol{b}_{T} \| \boldsymbol{P}_{h \perp}\right|\right)\left(\mathcal{F}_{U T, T}^{\sin \left(\phi_{h}-\phi_{S}\right)}+\varepsilon \mathcal{F}_{U T, L}^{\sin \left(\phi_{h}-\phi_{S}\right)}\right)\right. \\
& +\varepsilon \sin \left(\phi_{h}+\phi_{S}\right) J_{1}\left(\left|\boldsymbol{b}_{T}\right|\left|\boldsymbol{P}_{h \perp}\right|\right) \mathcal{F}_{U T}^{\sin \left(\phi_{h}+\phi_{S}\right)} \\
& +\varepsilon \sin \left(3 \phi_{h}-\phi_{S}\right) J_{3}\left(\left|\boldsymbol{b}_{T}\right|\left|\boldsymbol{P}_{h \perp}\right|\right) \mathcal{F}_{U T}^{\sin \left(3 \phi_{h}-\phi_{S}\right)} \\
& +\sqrt{2 \varepsilon(1+\varepsilon)} \sin \phi_{S} J_{1}\left(\left|\boldsymbol{b}_{T}\right|\left|\boldsymbol{P}_{h \perp}\right|\right) \mathcal{F}_{U T}^{\sin \phi_{S}} \\
& \left.+\sqrt{2 \varepsilon(1+\varepsilon)} \sin \left(2 \phi_{h}-\phi_{S}\right) J_{2}\left(\left|\boldsymbol{b}_{T} \| \boldsymbol{P}_{h \perp}\right|\right) \mathcal{F}_{U T}^{\sin \left(2 \phi_{h}-\phi_{S}\right)}\right] \\
& +\left|\boldsymbol{S}_{\perp}\right| \lambda_{e}\left[\sqrt{1-\varepsilon^{2}} \cos \left(\phi_{h}-\phi_{S}\right) J_{1}\left(\left|\boldsymbol{b}_{T}\right|\left|\boldsymbol{P}_{h \perp}\right|\right) \mathcal{F}_{L T}^{\cos \left(\phi_{h}-\phi_{S}\right)}\right. \\
& +\sqrt{2 \varepsilon(1-\varepsilon)} \cos \phi_{S} J_{0}\left(\left|\boldsymbol{b}_{T}\right|\left|\boldsymbol{P}_{h \perp}\right|\right) \mathcal{F}_{L T}^{\cos \phi_{S}} \\
& \left.\left.+\sqrt{2 \varepsilon(1-\varepsilon)} \cos \left(2 \phi_{h}-\phi_{S}\right) J_{2}\left(\left|\boldsymbol{b}_{T}\right|\left|\boldsymbol{P}_{h \perp}\right|\right) \mathcal{F}_{L T}^{\cos \left(2 \phi_{h}-\phi_{S}\right)}\right]\right\}
\end{aligned}
$$

where in the parton model framework the structure functions $\mathcal{F}_{X Y, Z}$ are now given as simple products of Fourier Transforms of TMDs. Here we consider the unpolarized and double longitudinal structure functions,

$$
\begin{aligned}
\mathcal{F}_{U U, T} & =x \sum_{a} e_{a}^{2} \tilde{f}_{1}^{a}\left(x, z^{2} \boldsymbol{b}_{T}^{2}\right) \tilde{D}_{1}^{a}\left(z, \boldsymbol{b}_{T}^{2}\right) \\
\mathcal{F}_{L L} & =x \sum_{a} e_{a}^{2} \tilde{g}_{1 L}^{a}\left(x, z^{2} \boldsymbol{b}_{T}^{2}\right) \tilde{D}_{1}^{a}\left(z, \boldsymbol{b}_{T}^{2}\right)
\end{aligned}
$$

where the Fourier transform of the TMDs are defined as

$$
\begin{aligned}
& \tilde{f}\left(x, \boldsymbol{b}_{T}^{2}\right)=\int d^{2} \boldsymbol{k}_{\perp} e^{i \boldsymbol{b}_{T} \cdot \boldsymbol{k}_{\perp}} f\left(x, \boldsymbol{k}_{\perp}^{2}\right)=2 \pi \int d \boldsymbol{k}_{\perp} \boldsymbol{k}_{\perp} J_{0}\left(\left|\boldsymbol{b}_{T}\right|\left|\boldsymbol{k}_{\perp}\right|\right) f\left(x, \boldsymbol{k}_{\perp}^{2}\right), \\
& \tilde{D}\left(z, \boldsymbol{b}_{T}^{2}\right)=\int d^{2} \boldsymbol{p}_{\perp} e^{i \boldsymbol{b}_{T} \cdot \boldsymbol{p}_{\perp}} D\left(z, \boldsymbol{p}_{\perp}^{2}\right)=2 \pi \int d \boldsymbol{p}_{\perp} \boldsymbol{p}_{\perp} J_{0}\left(\left|\boldsymbol{b}_{T}\right|\left|\boldsymbol{p}_{\perp}\right|\right) D\left(x, \boldsymbol{p}_{\perp}^{2}\right) .
\end{aligned}
$$

\subsection{Bessel weighting of experimental observables}

In this sub-section we introduce Bessel weighting of experimental observables, cross sections and asymmetries, based on the $b_{T}$ representation of the SIDIS cross section, eq. (2.4). In a partonic framework, "Bessel weighted experimental observables" are quantities which can 
be presented as simple products of Fourier transforms of distribution and fragmentation functions, allowing the application of standard flavor decomposition procedures. Here we will apply this technique to the double longitudinal spin asymmetry. From eq. (2.4) one can project out the unpolarized and double longitudinally polarized structure functions $\mathcal{F}_{L L}$, and $\mathcal{F}_{U U, T}$, by integrating with the zeroth order Bessel function $J_{0}\left(\left|\boldsymbol{b}_{T}\right|\left|\boldsymbol{P}_{h \perp}\right|\right)$ over the transverse momentum of the produced hadron $\boldsymbol{P}_{h \perp}$. We arrive at an expression for the longitudinally polarized cross section $\tilde{\sigma}^{ \pm}\left(b_{T}\right)$ in $\boldsymbol{b}_{T}$-space

$$
\tilde{\sigma}^{ \pm}\left(b_{T}\right)=2 \pi \int \frac{d \sigma^{ \pm}}{d \Phi} J_{0}\left(\left|\boldsymbol{b}_{T}\right|\left|\boldsymbol{P}_{h \perp}\right|\right) P_{h \perp} d P_{h \perp}
$$

where $d \Phi \equiv d x d y d \psi d z d P_{h \perp} P_{h \perp}$ represents shorthand notation for the phase space differential and $\left|\boldsymbol{b}_{T}\right| \equiv b_{T}$, and $\left|\boldsymbol{P}_{h \perp}\right| \equiv P_{h \perp}, d \sigma^{ \pm} / d \Phi$ is the differential cross section where \pm labels the double longitudinal spin combinations $S_{\|} \lambda_{e}= \pm 1$. Note that in our definition $b_{T}$ is the Fourier conjugate variable to $P_{h \perp}[39]$.

Now we form the double longitudinal spin asymmetry

$$
A_{L L}^{J_{0}\left(b_{T} P_{h \perp}\right)}\left(b_{T}\right)=\frac{\tilde{\sigma}^{+}\left(b_{T}\right)-\tilde{\sigma}^{-}\left(b_{T}\right)}{\tilde{\sigma}^{+}\left(b_{T}\right)+\tilde{\sigma}^{-}\left(b_{T}\right)} \equiv \frac{\tilde{\sigma}_{L L}\left(b_{T}\right)}{\tilde{\sigma}_{U U}\left(b_{T}\right)}=\sqrt{1-\varepsilon^{2}} \frac{\sum_{a} e_{a}^{2} \tilde{g}_{1 L}^{a}\left(x, z^{2} b_{T}^{2}\right) \tilde{D}_{1}^{a}\left(z, b_{T}^{2}\right)}{\sum_{a} e_{a}^{2} \tilde{f}_{1}^{a}\left(x, z^{2} b_{T}^{2}\right) \tilde{D}_{1}^{a}\left(z, b_{T}^{2}\right)} .
$$

The experimental procedure to study the structure functions in $b_{T}$-space amounts to discretizing the momentum phase space in eq. (2.9) and constructing the sums and differences of these discretized cross sections. The technical details of this procedure given in appendix A and B. Using these results, the double longitudinal spin asymmetry, eq. (2.10) results in an expression of sums and differences of Bessel functions for a given set of experimental events. The resulting expression for the spin asymmetry is

$$
A_{L L}^{J_{0}\left(b_{T} P_{h \perp}\right)}\left(b_{T}\right)=\frac{\sum_{j}^{N^{+}} J_{0}\left(b_{T} P_{h \perp j}^{[+]}\right)-\sum_{j}^{N^{-}} J_{0}\left(b_{T} P_{h \perp j}^{[-]}\right)}{\sum_{j}^{N^{+}} J_{0}\left(b_{T} P_{h \perp j}^{[+]}\right)+\sum_{j}^{N^{-}} J_{0}\left(b_{T} P_{h \perp j}^{[-]}\right)},
$$

where $j$ indicates a sum on \pm -helicity events, ${ }^{1}$ and where $N^{ \pm}$is the number of events with positive/negative products of lepton and nucleon helicities.

The cross sections $\tilde{\sigma}^{ \pm}\left(b_{T}\right)$ can be extracted for any given $b_{T}$ using sums over the same set of data. These cross sections contain the same information as the cross sections, $d \sigma / d \Phi$ in eq. (2.9) differential with respect to the outgoing hadron momentum. The momentum dependent and the $b_{T}$-dependent representations of the cross section are related by a $2-\mathrm{D}$ Fourier-transform in cylinder coordinates. eq. (2.11) and its generalization to other spin and azimuthal asymmetries provides another lever arm to study the partonic content of hadrons through the Bessel weighing procedure in Fourier $b_{T}$ space (see also $[57,58]$ ).

\footnotetext{
${ }^{1}$ Note, the + helicity and - helicity events are in two different, independent data sets of transverse momenta.
} 
In order to test the Bessel weighting of experimental observables for the double longitudinal spin asymmetry we will use a Monte Carlo generator which has been developed for the extraction of TMDs from SIDIS. In the next section we describe this new dedicated Monte Carlo generator which includes quark intrinsic transverse momentum within the generalized parton model.

\section{Fully differential Monte Carlo for SIDIS}

\subsection{The Monte Carlo and the generalized parton model}

A Monte Carlo generator is a crucial component in testing experimental procedures such as those described in eq. (2.11). In order to check the Bessel weighting technique we need a Monte Carlo that generates events in phase space with different TMD model inputs. It should also include explicit dependence on intrinsic parton transverse momentum $k_{\perp}$ and $p_{\perp}$. We reconstruct weighted asymmetries according to eq. (2.11), and in turn compare the generated events in momentum space which are then Fourier transformed. In keeping with the parton model picture however, a cross-section based on structure functions from eqs. (2.2) and (2.3) cannot be used for these purposes, since the simple parton model factorization would allow the MC generator to produce events that violate four-momentum conservation and thus are unphysical.

Therefore, the Monte Carlo generator we use has been developed to study partonic intrinsic motion using the framework of the so-called generalized parton model described in detail in ref. [29]. While including target mass corrections, more importantly for our study, it generates only events allowed by the available physical phase space.

In order to establish the proper kinematics of the phase space for the Monte Carlo consider the SIDIS process

$$
\ell(l)+N(P) \rightarrow \ell\left(l^{\prime}\right)+h\left(P_{h}\right)+X,
$$

where $\ell$ is the incident lepton, $N$ is the target nucleon, and $h$ represents the observed hadron, and the four-momenta are given in parenthesis. Following the Trento conventions [45], the spatial component of the virtual photon momentum $q$ is along the positive $z$ direction and the proton momentum $P$ is in the opposite direction, as depicted in figure 1. In the parton model, the virtual photon scatters off an on-shell quark where the initial quark momentum $k$, and scattered quark momentum $k^{\prime}$, have the same intrinsic transverse momentum component $\boldsymbol{k}_{\perp}$ with respect to the $z$ axis, and where the initial quark has the fraction $x$ of the proton momentum. The produced hadron momentum, $P_{h}$ has the fraction $z$ of scattered quark momentum $k^{\prime}$ in the $(\tilde{x}, \tilde{y}, \tilde{z})$ frame and $\boldsymbol{p}_{\perp}$ is the transverse momentum component with respect to the scattered quark $k^{\prime}$.

A great deal of phenomenological effort has been devoted to using the generalized parton model (see for example [29, 34, 59]), incorporating intrinsic quark transverse momentum, to account for experimentally observed spin and azimuthal asymmetries as a function of the produced hadron's transverse momentum $P_{h \perp}$ in SIDIS processes. In order to take into account non-trivial kinematic effects that are neglected from the standard 
parton model approximations [25, 27], such as discarding small momenta in the struck and fragmenting quarks, and discarding transverse momentum kinematic corrections due to hard scattering we develop a Monte Carlo based on the fully differential SIDIS cross section [29] which is given by,

$$
\begin{aligned}
& \frac{d \sigma}{d x d y d z d p_{\perp}^{2} d k_{\perp}^{2} d \phi_{l^{\prime}} d \phi_{k} d \tilde{\phi}}=\frac{1}{2} K(x, y) J\left(x, Q^{2}, \boldsymbol{k}_{\perp}^{2}\right) \\
& \quad \times x \sum_{a} e_{a}^{2}\left[f_{a}\left(x_{L C}, \boldsymbol{k}_{\perp}^{2}\right) D_{1, a}\left(z_{L C}, \boldsymbol{p}_{\perp}^{2}\right)+\lambda \sqrt{1-\varepsilon^{2}} g_{1 L, a}\left(x_{L C}, \boldsymbol{k}_{\perp}^{2}\right) D_{1, a}\left(z_{L C}, \boldsymbol{p}_{\perp}^{2}\right)\right],
\end{aligned}
$$

where the summation runs over quarks flavors and, $\lambda$ is the product of target polarization and beam helicity $(\lambda= \pm 1), \phi_{l^{\prime}}$ is the scattered lepton azimuthal angle, ${ }^{2}$ and

$$
K(x, y)=\frac{\alpha^{2}}{x y Q^{2}} \frac{y^{2}}{2(1-\varepsilon)}\left(1+\frac{\gamma^{2}}{2 x}\right), \quad \varepsilon=\frac{1-y-\frac{1}{4} \gamma^{2} y^{2}}{1-y+\frac{1}{2} y^{2}+\frac{1}{4} \gamma^{2} y^{2}},
$$

and the Jacobian $J$ is given by

$$
J\left(x, Q^{2}, \boldsymbol{k}_{\perp}^{2}\right)=\frac{x}{x_{L C}}\left(1+\frac{x^{2}}{x_{L C}^{2}} \frac{\boldsymbol{k}_{\perp}^{2}}{Q^{2}}\right)^{-1} .
$$

Here the cross section is "fully differential" in the transverse momentum of the target and fragmenting quark. This form of the cross section will allow us to implement the physical energy and momentum phase space constraints in the Monte Carlo generator. In order to calculate the cross-section in terms of observed momenta (only linear combinations of $\boldsymbol{k}_{\perp}$ and $\boldsymbol{p}_{\perp}$ can be measured experimentally) we need to integrate eq. (3.2) in $d^{2} \boldsymbol{k}_{\perp} d^{2} \boldsymbol{p}_{\perp}$ taking into account kinematical relations consistent with the observed final hadron momentum $P_{h \perp}$.

We elaborate further on the kinematics for the Monte Carlo generator. In above equations $x$ is the Bjorken variable, while $x_{L C}=k^{-} / P^{-}$is the light-cone (LC) fraction of the proton momentum carried by the quark $k$ [29]. The quark four momentum is given by,

$$
\begin{aligned}
k_{0} & =x_{L C} P^{\prime}+\frac{k_{\perp}^{2}}{4 x_{L C} P^{\prime}}, \\
k_{x}=k_{\perp} \cos \left(\phi_{k}\right), \quad k_{y} & =k_{\perp} \sin \left(\phi_{k}\right), \quad k_{z}=-x_{L C} P^{\prime}+\frac{k_{\perp}^{2}}{4 x_{L C} P^{\prime}},
\end{aligned}
$$

where $k_{0}$ is the quark energy, and $k_{\{x, y, z\}}$ are the $x, y$ and $z$ components of the quark momentum in the center of mass (CM) frame of virtual photon and proton, and $P^{\prime} \equiv$ $0.5\left(E_{p}+\left|P_{p z}\right|\right)$, where the proton energy in the CM is $E_{p}=\sqrt{P_{p z}^{2}+M^{2}}$. Taking into account the nucleon mass, and the on-shell condition for the intial quark, the following expressions for $x_{L C}=k^{+} / P^{+}$and the Nachtman variable $x_{N}$ become

$$
x_{L C}=\frac{x}{x_{N}}\left(1+\sqrt{1+\frac{4 k_{\perp}^{2}}{Q^{2}}}\right), \quad x_{N}=1+\sqrt{1+\frac{4 M^{2} x^{2}}{Q^{2}}},
$$

\footnotetext{
${ }^{2}$ Integration over $\phi_{l^{\prime}}$ gives $2 \pi$, since everything is symmetric along beam direction, although we need to keep it for further analysis, when one reconstructs generated events in the real experimental setup.
} 


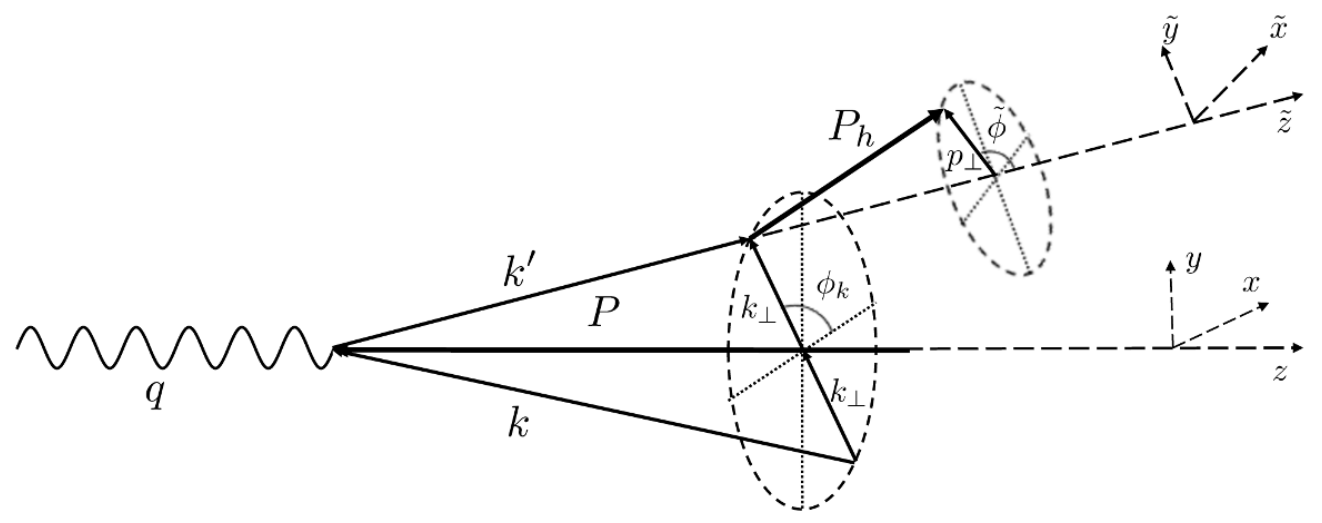

Figure 1. Kinematics of the process. $q$ is the virtual photon, $k$ and $k^{\prime}$ are the initial and struck quarks, $k_{\perp}$ is the quark transverse component. $P_{h}$ is the final hadron with a $p_{\perp}$ component, transverse with respect to the fragmenting quark $k^{\prime}$ direction.

where $k_{\perp}=\left|\boldsymbol{k}_{\perp}\right|$ is the parton transverse momentum. The scattered quark momentum $k^{\prime}$ is constructed using $k^{\prime}=k+q$ (see figure 1). Further, $\phi_{k}$ is the initial quark azimuthal angle, $z_{L C}=P_{h}^{+} / k^{+}$is the light-cone fraction of the quark momentum carried by the resulting hadron in the $(\tilde{x}, \tilde{y}, \tilde{z})$-system [29], where $\tilde{z}$ is aligned along the scattered quark $k^{\prime}$. The final hadron momentum is constructed using,

$$
P_{h \tilde{x}}=p_{\perp} \cos (\tilde{\phi}), \quad P_{h \tilde{y}}=p_{\perp} \sin (\tilde{\phi}), \quad P_{h \tilde{z}}=z_{L C} k_{0}^{\prime}-\frac{p_{\perp}^{2}+M_{h}^{2}}{4 z_{L C} k_{0}^{\prime}}
$$

where $\tilde{\phi}$ is the angle between quark and hadron planes, and $\phi_{h}$ is the angle between leptonic and hadronic planes according to the Trento convention and $P_{h \perp}$ is the final hadron transverse momentum [45]. The final hadron SIDIS variables $\phi_{h}, P_{h \perp}$ and $z$ are calculated after event generation. Here we should note, that theoretical or phenomenological distribution and fragmentation functions are expected to be in the light cone coordinate system (see eq. (3.2)). Motivated by the fact that $x_{L C} \simeq x$ and $z_{L C} \simeq z$ is a widely used approximation in global fitting, the unpolarized and helicity TMDs are then $f_{1, q}\left(x, \boldsymbol{k}_{\perp}^{2}\right)$ and $g_{1 L, q}\left(x, \boldsymbol{k}_{\perp}^{2}\right)$, and $D_{1, q}\left(z, \boldsymbol{p}_{\perp}^{2}\right)$ is the unpolarized fragmentation function. In our Monte Carlo generator we adopt the parton kinematics in $[29,60]$ with the additional requirements, that the kinematics of the initial and final parton momenta are kept exact [61], and the nucleon mass is not set to zero.

Finally we note, the Jacobian becomes unity if $k_{\perp}^{2} / Q^{2}$ corrections are neglected and thus, the usual parton model expression can be recovered in this approximation from eq. (3.2).

An interesting question concerns the validity of the the parton model and the generalized parton model at the relatively low beam energies available in experiments today. The parton model is an approximation that assumes certain components of the intrinsic parton momenta are suppressed for large beam energies and can thus be integrated out from the distributions. This becomes apparent in eqs. (2.2) and (2.3), where the delta function in the \pm components of parton momenta decouple from transverse momentum resulting in a 
delta function in only the two transverse dimensions. An explicit four-momentum conservation law embedded in the formula of the cross section is thus lost. A particularly striking consequence that one observes is that there is no explicit mechanism that prevents events at values of $P_{h \perp}$ larger than allowed by the finite beam energy. Naturally, the lower the beam energy becomes, the more serious the inaccuracies of the parton model have to be taken. On the other hand, the "fully differential" cross section eq. (3.2) of the generalized parton model allows us to include in our Monte Carlo both transverse momentum and the physical energy and momentum phase space constraints. We used the widely accepted parton model approximation of setting the initial parton on-shell (assumption that virtual photon interacts with an on-mass shell quark). ${ }^{3}$ But it is important to emphasize that the approximations we have made, which are consistent with a generalized parton model framework, enable us to implement a Monte Carlo that incorporates the correct phase space momentum constraints and satisfies the requirements we outlined in this section.

Thus, our Monte Carlo simulation allows us to take the factorized form of the generalized parton model cross section eq. (3.2) as a basis and then to impose four-momentum conservation for the partons according to figure 1, assuming the initial quark is on-shell with non-zero mass. We also take a non-zero target mass into account. This procedure does not necessarily lead to a more accurate description of the underlying physics, because it still rests on the simplified picture of the generalized parton model and involves the approximation of an on-shell quark. Nonetheless, implementing these modifications can give us an indication for the magnitude of the uncertainties resulting from the aforementioned kinematic approximations in the parton model.

Note that our goal is to study the applicability of Bessel weighting to experimental data, for which we explicitly need $k_{\perp}$ and $p_{\perp}$ dependences in the Monte Carlo generator. Alongside with this goal it is interesting to investigate how well the approximations of the simple parton model are justified in the current relatively low energy experimental set-up. One would expect that if approximations that lead to the parton model expressions for structure functions are justified, then the generalized parton model expression would not spoil this approximation numerically. On the other hand if the generalized parton model gives notably different results with respect to a naive parton model, one would expect that kinematics of the experiment does not allow a certain type of approximations and the theoretical/phenomenological description should be improved.

Ultimately the comparison with experimental data will allow us to address these questions. In the mean time in section 3.2 we will study some of these issues using our Monte Carlo generator based on the generalized parton model. This will allow us to explore the validity of certain kinematical approximations and also to understand how parameters of the implemented distributions are different from extracted distributions. Once we have control over these issues in the kinematics of low energy experiments we will also compare in section 3.3, our results with data from HERMES experiment as an illustration of possible effects. The applicability of the Bessel weighting technique and resulting uncertainties is a separate issue and will be addressed in section 4 .

\footnotetext{
${ }^{3}$ The confined quark has a non-zero virtuality. Such effects in Monte Carlo generators have been studied in ref. [62].
} 
In the Monte Carlo generator software, we used the general-purpose, self-adapting event generator, Foam [63], for drawing random points according to an arbitrary, userdefined distribution in $n$-dimensional space.

\subsection{Kinematical distributions}

Implementing the Monte Carlo, we generate kinematical distributions in $x, z, k_{\perp}$, and $p_{\perp}$ of SIDIS events for several model inputs of TMDs. These distributions are then used to check the consistency of dependence of extracted quantities under different model assumptions, including, for example Gaussian and non-Gaussian distributions in transverse momentum.

In case the dependence is assumed to be a Gaussian, $x$ and $z$ dependent widths are assumed, so that TMDs take the following form,

$$
\begin{aligned}
f_{1}\left(x, \boldsymbol{k}_{\perp}^{2}\right) & =f_{1}(x) \frac{1}{\left\langle k_{\perp}^{2}(x)\right\rangle_{f_{1}}} \exp \left(-\frac{\boldsymbol{k}_{\perp}^{2}}{\left\langle k_{\perp}^{2}(x)\right\rangle_{f_{1}}}\right), \\
g_{1 L}\left(x, \boldsymbol{k}_{\perp}^{2}\right) & =g_{1 L}(x) \frac{1}{\left\langle k_{\perp}^{2}(x)\right\rangle_{g_{1}}} \exp \left(-\frac{\boldsymbol{k}_{\perp}^{2}}{\left\langle k_{\perp}^{2}(x)\right\rangle_{g_{1}}}\right), \\
D_{1}\left(z, \boldsymbol{p}_{\perp}^{2}\right) & =D_{1}(z) \frac{1}{\left\langle p_{\perp}^{2}(z)\right\rangle} \exp \left(-\frac{\boldsymbol{p}_{\perp}^{2}}{\left\langle p_{\perp}^{2}(z)\right\rangle}\right),
\end{aligned}
$$

where $f(x)$ and $D(z)$ are corresponding collinear parton distribution and fragmentation functions and the widths are $x$ and $z$ dependent functions. In our studies we adopt the modified Gaussian distribution functions and fragmentation functions from eq. (3.9)-(3.11), in which $x$ and $k_{\perp}$ dependencies are inspired by AdS/QCD results [64, 65], with $\left\langle k_{\perp}^{2}(x)\right\rangle=$ $C x(1-x)$ and $\left\langle p_{\perp}^{2}(z)\right\rangle=D z(1-z)$, where the constants $C$ and $D$ may be different for different flavors and polarization states (see for example [38]). Similarly such non-factorized $x, k_{\perp}$ distribution functions are also suggested by the diquark spectator model [66] and the NJL-jet model $[36,67]$.

For the $x$ and $z$ dependence in eqs. (3.9) and (3.11) we use the parametrizations, $f_{1}(x)=(1-x)^{3} x^{-1.313}, g_{1 L}(x)=f_{1}(x) x^{0.7}$, and $D_{1}(z)=0.8(1-z)^{2}$, using input values $C=0.54 \mathrm{GeV}^{2}$ and $D=0.5 \mathrm{GeV}^{2}$. We also assume that $\left\langle k_{\perp}^{2}\right\rangle_{g_{1 L}}=0.8\left\langle k_{\perp}^{2}\right\rangle_{f_{1}}$; this assumption is consistent with lattice studies [54] and experimental measurements [14].

As an example of a non-Gaussian $k_{\perp}$ distribution we implement the following one inspired by the shape of the resulting distribution in the light-cone quark model $[68,69]$

$$
f_{1}\left(x, \boldsymbol{k}_{\perp}^{2}\right)=f_{1}(x) /\left(1+20.82 k_{\perp}^{2}+126.7 k_{\perp}^{4}+1285 k_{\perp}^{6}\right) .
$$

where the coefficients for $g_{1 L}\left(x, \boldsymbol{k}_{\perp}^{2}\right)$ are chosen such that effectively $\left\langle k_{\perp}^{2}\right\rangle_{g_{1 L}} /\left\langle k_{\perp}^{2}\right\rangle_{f_{1}}=0.8$.

We then generate events using the cross section from eq. (3.2) for both Gaussian and non-Gaussian initial distributions respectively, and we display the resulting transverse momentum distributions in figures 2 and 3. Note (as stated earlier) that the generator we construct is implemented with on-shell initial partons with four momentum conservation imposed. While this choice is not compulsory we adopt it as it allows us to fully reconstruct kinematics for a given event. At the same time, the limitations due to available phase space integration will modify the reconstructed distributions with respect to the input 


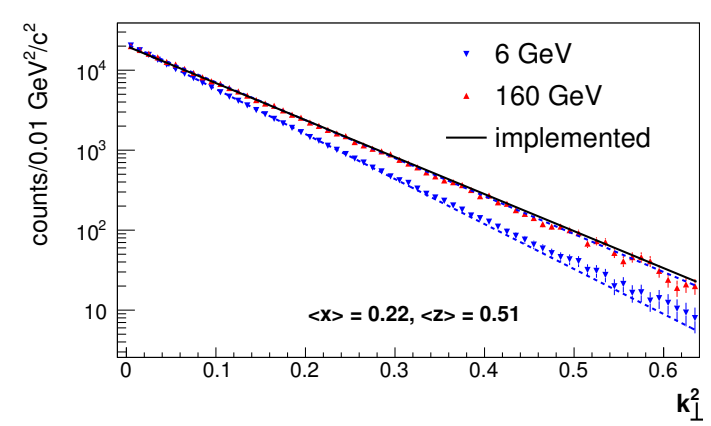

Figure 2. (Color online) The solid line is the Gaussian input distribution implemented using eq. (3.9), with red triangles coming from the Monte Carlo at $160 \mathrm{GeV}$ initial lepton energy, blue triangles coming from the Monte Carlo at $6 \mathrm{GeV}$. The dashed line represents the fit to the Monte Carlo distributions which returned values of $C=0.527 \mathrm{GeV}^{2}$ and $C=0.444 \mathrm{GeV}^{2}$ at $160 \mathrm{GeV}$ and $6 \mathrm{GeV}$ respectively.

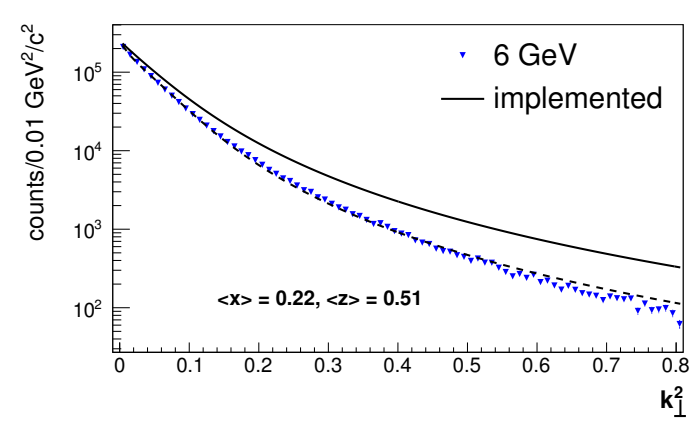

Figure 3. (Color online) The solid line is the implemented non-Gaussian distribution using eq. (3.12), with $\left\langle k_{\perp}^{2}\right\rangle=0.084 \mathrm{GeV}^{2}$, and the dashed curve represents the fit to the Monte Carlo distribution with the value of $\left\langle k_{\perp}^{2}\right\rangle=$ $0.064 \mathrm{GeV}^{2}$ at $6 \mathrm{GeV}$ initial lepton beam energy. The available phase space dictated by four momentum conservation results in a deformation of the input distribution.

distributions. We analyze the effect of the available phase space in the Monte Carlo on the average $\left\langle k_{\perp}^{2}\right\rangle$ for finite beam energies as a function of $x$ by calculating the effective $\left\langle k_{\perp}^{2}\right\rangle$ from the following formula,

$$
\left\langle k_{\perp}^{2}(x)\right\rangle=\frac{\int d^{2} \boldsymbol{k}_{\perp} k_{\perp}^{2} d \sigma_{M C}}{\int d^{2} \boldsymbol{k}_{\perp} d \sigma_{M C}}=\frac{\sum_{j=1}^{N} k_{\perp j}^{2}}{N},
$$

where the index $j$ runs over the $N$ Monte Carlo generated events. Note, $d \sigma_{M C}$ is the cross section of the Monte Carlo simulation, that is eq. (3.2), modified by imposing the four momenta conservation and on-shell condition for initial quark.

Indeed in figures 2 and 3 we find when comparing the Monte Carlo generated events with the input distributions, using eq. (3.9) and eq. (3.12), shown as solid black curves for a given $x$, that the larger $k_{\perp}$ values of the Monte Carlo events (red triangles up, $160 \mathrm{GeV}$ beam energy, and blue triangles down, $6 \mathrm{GeV}$ beam energy) are suppressed due to the available phase space imposed by both the finite beam energy, and four momentum conservation in the Monte Carlo. The fit of the Monte Carlo distributions for the modified Gaussian model are shown as dashed lines displayed in figure 2. They return the fitted values $C=0.527 \mathrm{GeV}^{2}$ and $C=0.444 \mathrm{GeV}^{2}$ for the $160 \mathrm{GeV}$ and $6 \mathrm{GeV}$ Monte Carlo simulations respectively. In figure 3 we study the effect of the non-Gaussian distribution eq. (3.12). Integrating eq. (3.13) over $k_{\perp}$ gives a value of $\left\langle k_{\perp}^{2}\right\rangle=0.084 \mathrm{GeV}^{2}$, and the dashed curve represents the fit to the Monte Carlo distribution with a value of $\left\langle k_{\perp}^{2}\right\rangle=0.064 \mathrm{GeV}^{2}$ for the $6 \mathrm{GeV}$ initial lepton beam energy.

In figure 4 . the average $\left\langle k_{\perp}^{2}\right\rangle$ versus $x$ from the Monte Carlo for different incoming beam energies, for $0.5<z<0.52$, is presented. For the modified Gaussian distribution function 


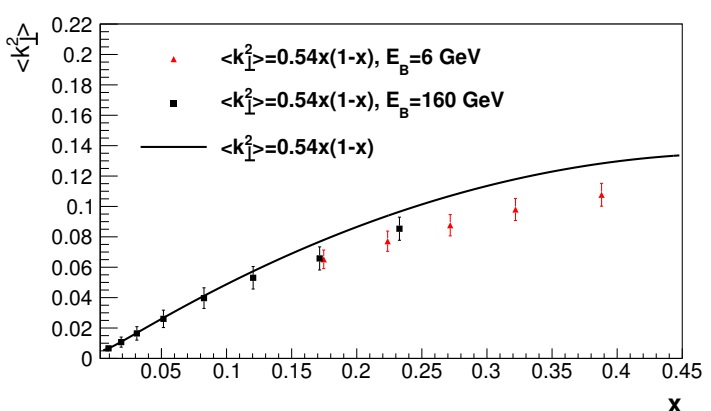

Figure 4. (Color online) $\left\langle k_{\perp}^{2}(x)\right\rangle$ versus $x$ for the unpolarized $\left(d \sigma^{+}+d \sigma^{-}\right)$cross-section for $0.50<z<0.52$, for two Monte Carlo runs with beam energies $6 \mathrm{GeV}$ and $160 \mathrm{GeV}$, with the modified Gaussian distribution function and fragmentation functions. The solid line represents the input function, while the Monte Carlo generated values are black squares for $160 \mathrm{GeV}$ and red triangles for $6 \mathrm{GeV}$.

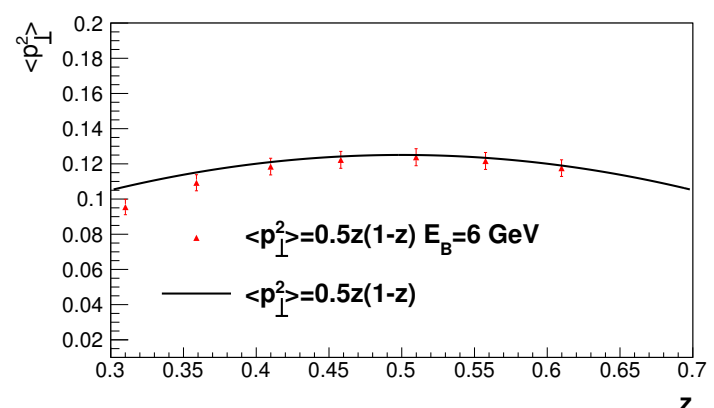

Figure 5. (Color online) $\left\langle p_{\perp}^{2}(z)\right\rangle$ versus $z$ for the unpolarized $\left(d \sigma^{+}+d \sigma^{-}\right)$cross-section for the $6 \mathrm{GeV}$ beam for $0.20<x<0.25$ from the Monte Carlo with the modified Gaussian distribution and fragmentation functions as compared to the analytic result using eq. (3.2) and the input distributions. The solid line represents the input function, while the Monte Carlo generated values are the red triangles for $6 \mathrm{GeV}$.

with the input value $\left\langle k_{\perp}^{2}(x)\right\rangle=0.54 x(1-x) \mathrm{GeV}^{2}$, the suppression of the generated $\left\langle k_{\perp}^{2}(x)\right\rangle$ compared to input distributions (solid line) is greater for the lower beam energy. In figure 5 the constraints of four momentum conservation also affect the $p_{\perp}^{2}$ distributions, which in turn also affect the observed $P_{h \perp}$ distribution.

The systematic deformation of the extraction of the TMDs in momentum space due to the kinematic constraints has been studied in detail using our fully differential Monte Carlo. We conclude this section with the general observation that imposing four momentum conservation in the event generator effectively modifies the initial distributions due to the limitations of the available phase space in the generator. This deformation is more pronounced at lower energies or $Q^{2}$. A shift of a few percent is visible for $160 \mathrm{GeV}$ incoming lepton beam energy, while for the lower $6 \mathrm{GeV}$ energy the effective $\left\langle k_{\perp}^{2}\right\rangle$ is lower than the input value by approximately $\sim 20 \%$.

At this point let us comment on the applicability of the description of experimental data in terms of a simple parton model. As we can see from figures 2 , and 4 , the results are consistent for large energy $(160 \mathrm{GeV})$ while they exhibit a significant shift at lower energy $(6 \mathrm{GeV})$ for the same input parameters. In fact the kinematical corrections due to imposing four-momentum conservation and target mass corrections, grow at lower energies as one would expect. At the same time, a $20 \%$ correction is well within the expected accuracy of the parton model approximation; remember that one usually neglects $k_{\perp}^{2} / Q^{2}$ corrections, and one would expect that after inclusion of such corrections one can achieve a better quantitative description of the data. In the next section we present the outcome of the Monte Carlo compared to experimental data from HERMES, and discuss its relevance. 


\subsection{The Cahn effect in the Monte Carlo generator}

As an example of an application of our constructed Monte Carlo we present a study of the Cahn effect $[40,41]$ contribution to the average $\langle\cos \phi\rangle$ moment in SIDIS. We generate Monte Carlo events using the following expression for the cross section [29],

$$
\frac{d \sigma}{d x d y d z d^{2} \boldsymbol{p}_{\perp} d^{2} \boldsymbol{k}_{\perp}}=K(x, y) J\left(x, Q^{2}, \boldsymbol{k}_{\perp}^{2}\right) \sum_{a} f_{1, a}\left(x, \boldsymbol{k}_{\perp}^{2}\right) D_{1, a}\left(z, \boldsymbol{p}_{\perp}^{2}\right) \frac{\hat{s}^{2}+\hat{u}^{2}}{Q^{4}}
$$

where $\hat{s}=(l+k)^{2}$ and $\hat{u}=\left(k-l^{\prime}\right)^{2}$ (see figure 1). As stated above, in the Monte Carlo we impose four momentum conservation with target mass corrections.

In figure 6 we present output from the Monte Carlo using the non-factorized Gaussian distribution function and fragmentation function (eqs. (3.9) and (3.11)). We also compare our results to the HERMES data [70], and ref. [60]. The dashed line in figure 6 represents the naive parton model result without any kinematical constraint on parton momenta while the solid line results from performing the computation with the kinematical constraints. One can see that taking into account these constraints is important for a description of the experimental data within this model. It is clear that the results of our Monte Carlo are comparable to that of [60] and close to HERMES data [70]. For the red triangles, we used $\left\langle k_{\perp}^{2}\right\rangle=0.54 x(1-x)$ and $\left\langle p_{\perp}^{2}\right\rangle=0.5 z(1-z) \mathrm{GeV}^{2}$. As one can see for HERMES kinematics the modified Gaussian TMDs reduces the contribution of the Cahn effect contribution to the $\left\langle\cos \left(\phi_{h}\right)\right\rangle$ moment. In ref. [60] this effect is achieved by imposing a so-called direction cut (that the quark moves in the forward direction with respect of the proton). In this Monte Carlo there are two main factors that modify the distribution; the four-momentum conservation and $x(z)$ dependent values of $\left\langle k_{\perp}^{2}\right\rangle\left(\left\langle p_{\perp}^{2}\right\rangle\right)$. One might expect that the Jacobian in eq. (3.14) plays a major role modifying transverse shape of resulting cross section, however we checked that it is not the case. The most important effect comes from taking into account kinematical constraints on parton momenta. One would conclude that taking these corrections into account is important for reliable analysis of experimental data.

In the next section we apply the Bessel weighting formalism for the double longitudinal spin asymmetry in semi-inclusive deep inelastic scattering to data from our Monte Carlo generator.

\section{Bessel weighted double spin asymmetry}

In this section, we present an extraction of the Bessel weighted double longitudinal spin asymmetry in $b_{T}$ space. We also carry out a study of the accuracy of such an extraction. We use the dedicated fully differential SIDIS single hadron Monte Carlo to generate events based on the input TMDs. For simplicity we perform this comparison in a one flavor approximation.

\subsection{Results from the Monte Carlo}

The Monte Carlo generated events are used like experimental events to extract both the Bessel weighted asymmetry $A_{L L}^{J_{0}\left(b_{T} P_{h \perp}\right)}$, and the ratio of the Fourier transform of $g_{1 L}$ to $f_{1}$, 


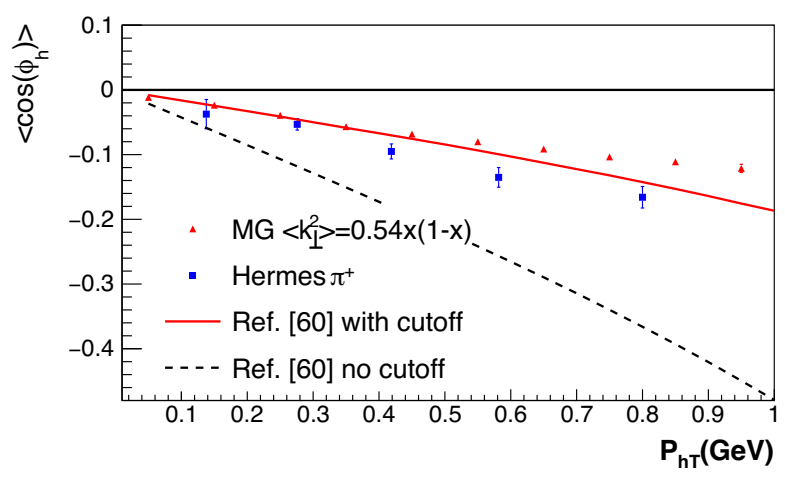

Figure 6. (Color online) The Cahn contribution in $\left\langle\cos \left(\phi_{h}\right)\right\rangle$ for $\pi^{+}$from the modified Gaussian (red triangles denoted as MG in the figure) PDFs using eq. (3.9) is presented for HERMES kinematics in comparison with ref. [60] (red solid and black dashed lines) and published HERMES data [70] (blue squares).

using the Bessel weighting method described in [39]. The results are then compared to the Monte Carlo input. The Bessel moments are extracted from the Monte Carlo with $6 \mathrm{GeV}$ beam energy using both the modified Gaussian type of functions (see eqs. (3.9)-(3.11)) and power law-tail like function (see eq. (3.12)).

The numerical results of our studies are summarized and displayed in figures 7 and 8 for the modified Gaussian distribution function and for the power law-tail like distribution function inputs respectively. In the left panel of figure 7 we show the Bessel-weighted asymmetry versus $b_{T}$. The blue curve labeled "BW Input", is the asymmetry calculated analytically using the right hand side of eq. (2.10) and the Fourier transformed input distribution functions (one can compare this with the model calculation in ref. [71]).

We now compare various distributions generated from the Monte Carlo. We plot the generated distribution using eq. (2.11) (full red points) labeled "BW $\left(P_{h \perp}\right)$ Generated", and the black triangles labeled "BW $\left(P_{h \perp}\right) \mathrm{Sm}+\mathrm{Acc}$ ", which represents the same extraction after experimental smearing and acceptance. For this we use the CLAS spectrometer [72], which is a quasi- $4 \pi$ detector, comprised of six azimuthally symmetric detector arrays, and uses a toroidal field to bend charged particles. Particle momenta and scattering angles were measured with a drift chamber tracking system with a relative accuracy of $0.3 \%$ to $2 \%$ in momentum, and about $3 \mathrm{mr}$ in angle and with less than $1 \%$ momentum resolution in the presented bin $\langle x\rangle=0.22$, and $\langle z\rangle=0.51$.

Next we consider the Fourier transform ratio $\tilde{g}_{1 L}$ to $\tilde{f}_{1}$, the (green) curve with triangles up labeled "BW $\left(k_{\perp}\right)$ " obtained from numerically Fourier transforming the $k_{\perp}$ distributions from the Monte Carlo generator on an event by event basis (see eq. (2.7)),

$$
\sqrt{1-\varepsilon^{2}} \frac{\tilde{g}_{1 L}\left(b_{T}\right)}{\tilde{f}_{1}\left(b_{T}\right)}=\frac{\sum_{j}^{N^{+}} J_{0}\left(b_{T} k_{\perp j}^{[+]}\right)-\sum_{j}^{N^{-}} J_{0}\left(b_{T} k_{\perp j}^{[-]}\right)}{\sum_{j}^{N^{+}} J_{0}\left(b_{T} k_{\perp j}^{[+]}\right)+\sum_{j}^{N^{-}} J_{0}\left(b_{T} k_{\perp j}^{[-]}\right)} .
$$



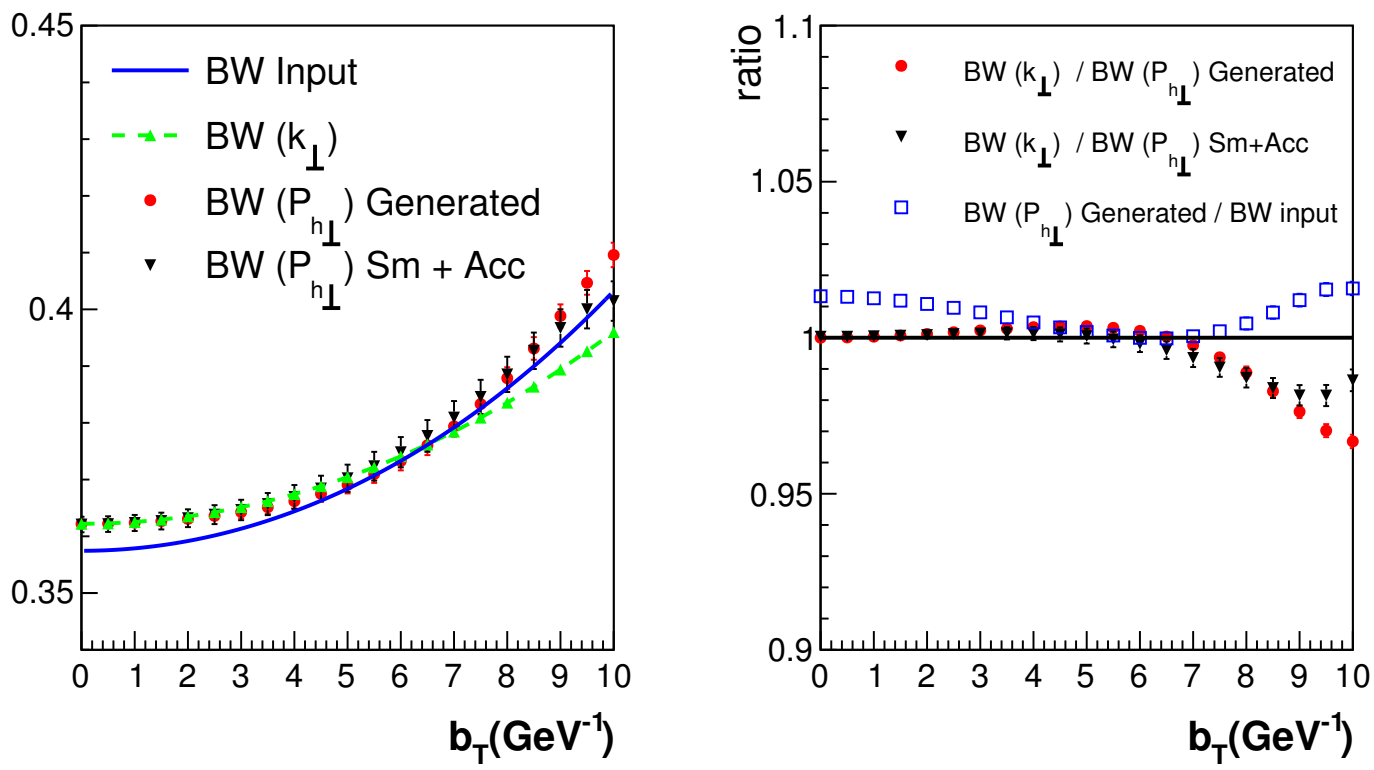

Figure 7. (Color online) Left panel: the ratio of Fourier transforms $\tilde{g}_{1 L} / \tilde{f}_{1}$ and the Bessel weighted asymmetry $A_{L L}^{J_{0}\left(b_{T} P_{h \perp}\right)}$ plotted versus $b_{T}$. The solid curve (blue) is the Fourier transform of the input to the Monte Carlo given by eq. (2.10), the red points are generated Monte Carlo events using eq. (2.11), and triangles down (black) represent results of Monte Carlo events after experimental smearing and acceptance at $\langle x\rangle=0.22$, and $\langle z\rangle=0.51$. The triangles up with dashed curve (green) are results of the Monte Carlo without inclusion of fragmentation functions (see text for discussion of errors). Right panel: ratios that represent the accuracy of our results.
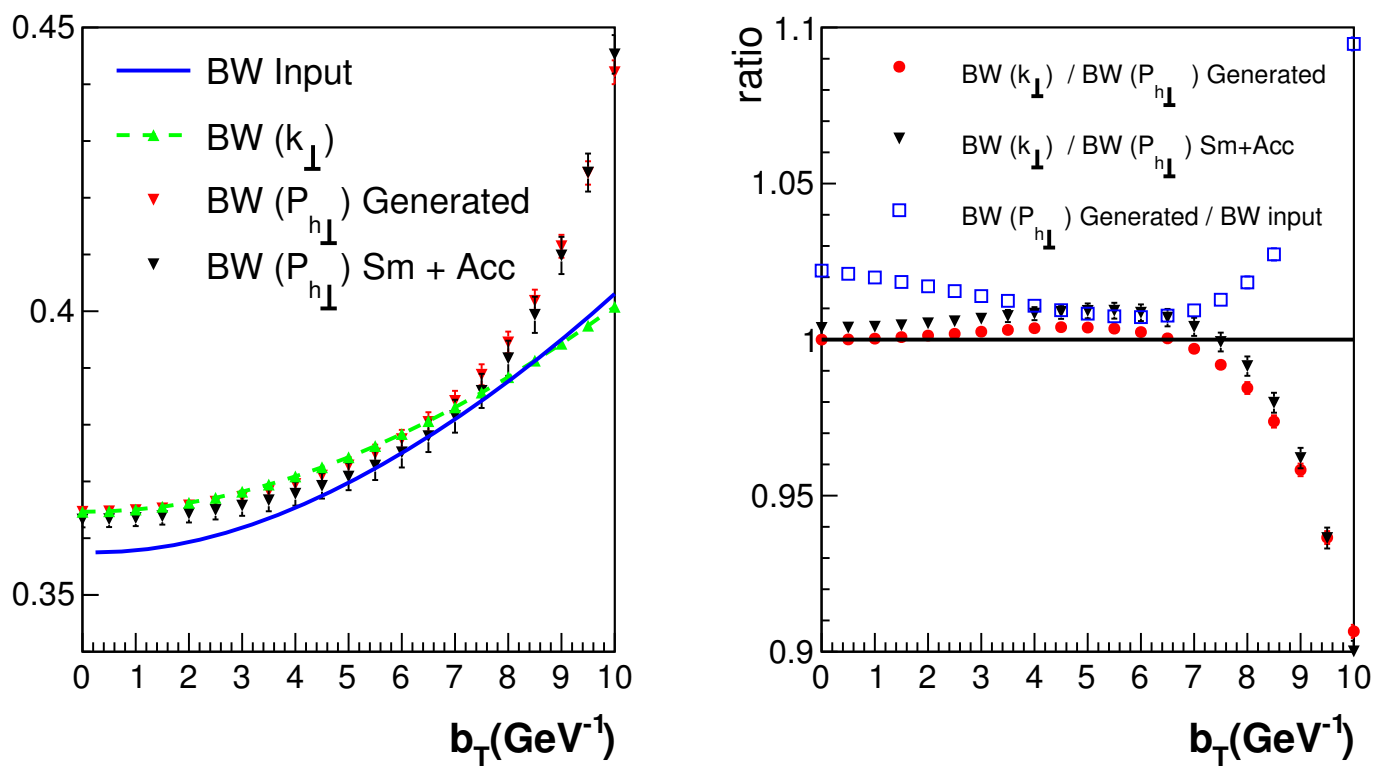

Figure 8. (Color online) The same as in figure 7 but here from the power-law tail distribution function based on the Monte Carlo (see text for discussion of errors). 
This quantity corresponds to the right hand side of eq. (2.10) in the one flavor approximation, where the fragmentation functions are expected to cancel out. For the Monte Carlo generated events, this cancellation is only an approximation, leading to the deviation between the red (or black) points and the green curve at large $b_{T}$. The reasons for the imperfect cancellation are discussed in section 4.2.

In order to quantitatively assess the deviation between the curves in the left panel of figure 7, we plot ratios of these values (see right panel). The red points represent the deviation from unity that is due to the imperfect cancellation of the fragmentation function. The black triangles represent the same after experimental smearing and acceptance are taken into account. Finally the open blue squares represent the deviation between the analytic result from the input distributions and the Monte Carlo generated events, eq. (2.11).

The error bars in $b_{T}$ space for each point give the statistical standard deviation. For each $b_{T}$ point, the statistical error bars are calculated from $\sim 250 \mathrm{k}$ independently generated events in momentum space (see appendix B for more details on error calculations). However, if we use the same data set to integrate over $P_{h \perp}$ for all $b_{T}$ points, the errors in $b_{T}$ space are correlated, which needs to be taken into account in the error analysis of any global fit to the data points. To circumvent this problem, we used different Monte Carlo samples for each $b_{T}$-point. In our numerical simulation we can afford to do that, because we can generate events copioulsy.

The same idea is applicable for future experiments at Jefferson Lab 12 [1], RHIC Spin [73] and Electron Ion Collider [5] that will deliver events in great abundance. In that case, one can divide the data into subsets, and take independent subsets of data for each value of $b_{T}$ to calculate the asymmetry. As a rule of thumb, if an experimental analysis can afford to have 5-10 bins in $P_{h \perp}$, then we expect there to be enough data to split it up for independent analyses at 5-10 values of $b_{T}$. However, if events are scarce, we need to find a way to carry out the error analysis using the same dataset for all values of $b_{T}$. An obvious way to do that might be to perform a generalized least squares fit based on the correlation/covariance matrix. Be aware, however, that using the inverse sample covariance matrix in a fit can be an unstable approach, for two reasons. First of all, the sample covariance matrix becomes a poor estimate of the true covariance matrix when the number of fit points is not many times smaller than the number of experimental observations, i.e., $N^{ \pm}$. In particular, the sample covariance matrix may be (close to) singular. Secondly, any systematic effects in the data that have been neglected in the model of the fit function may be amplified strongly by the inverse covariance matrix. Therefore, it often turns out to be more practical and stable to perform a weighted least squares fit, ignoring all correlations. This is a valid approach as long as we do not ignore the correlations in the subsequent error analysis of the resulting fit parameters. Resampling techniques provide simple yet powerful solutions to perform the correlated error analysis and are very popular in lattice QCD. We can take advantage of them in our situation as well. As explained in greater detail in appendix B and C, the sums $\tilde{S}^{ \pm}=\sum_{j}^{N^{ \pm}} J_{0}\left(b_{T} P_{h \perp j}^{[ \pm]}\right)$in eq. (2.11), (A.11) follow so-called compound Poisson distributions [74]. Therefore, we can make use of the variant of the bootstrap method proposed in ref. [75], which we describe in the context of Bessel weighted asymmetries in appendix $\mathrm{C}$. 


\subsection{Interpretation of the results}

One primary question addressed in this study is how robust the Bessel-weighting technique behaves under simulating real experimental conditions. Comparing the round (red) data points with the triangular (black) ones in figures 7 and 8, we see that switching on experimental smearing and acceptance in our simulation does not change the results significantly. Analyzing our MC results with four momenta conservation and target mass correction, we are able to distinguish two effects in the left panels of figures 7 and 8:

1. Solid (blue) curve versus triangular (green) data points: the distributions realized in the $M C$ simulation differ from the input distributions. In the $\mathrm{MC}$, the four-momentum conservation does not allow the variables $\boldsymbol{k}_{\perp}$ and $\boldsymbol{p}_{\perp}$ in eq. (3.2) to be sampled independently over the whole integration range, as it would have to be done to reproduce the unmodified parton model eqs. (2.2) and (2.3). The actual $\boldsymbol{k}_{\perp}$ and $\boldsymbol{p}_{\perp}$ distributions realized by the MC differ from the analytic input distributions eqs. (3.9)-(3.12) noticeably, especially in their widths. This has already been observed in figure 2 . The solid (blue) curve in the left panel of figures 7 and 8 is calculated from the input distributions according to the parton model; the FFs on the right hand side of eq. (2.10) cancel exactly in the single flavor scenario. Thus the solid curve can be compared to the triangle shaped (green) data points, which have also been calculated from a ratio of TMD PDFs, eq. (4.1), albeit with the actual distributions realized in the MC.

2. Triangular (green) data points vs. circular (red) data points: inadequacy of the generalized parton model to describe the data. In a single flavor scenario, the distribution functions $\tilde{D}_{1}^{a}$ cancel exactly on the right hand side of eq. (2.10). Therefore, there should not be any difference between the full asymmetry $A_{L L}^{J_{0}\left(b_{T} P_{h \perp}\right)}\left(b_{T}\right)$ of eqs. (2.10), (2.11) and the ratio of TMD PDFs eq. (4.1). However, we do observe a difference between the circular (red) data points and the triangular (green) data points in the left panels of figures 7 and 8. Again, the four-momentum conservation we have implemented is the reason for the observed difference. Since $\boldsymbol{k}_{\perp}$ and $\boldsymbol{p}_{\perp}$ are no longer sampled in accordance with eqs. (2.2) and (2.3), the right hand side of eq. (2.10) then needs modification. ${ }^{4}$ Therefore, we see only an incomplete cancellation of FFs for the Monte Carlo events.

To an experimentalist who is concerned about systematic errors attributed to the observables he or she extracts, the first of the two effects above is not an issue. The purpose of the generalized parton model is to provide a parametrization of the data one observes. Any effect of the underlying scattering mechanism that can be absorbed into the distributions does not contradict the validity of the model. The only concern one might have is that the distributions become beam energy $/ Q^{2}$ dependent, an issue that should be addressed using TMD evolution equations.

On the other hand, the second effect presented above can be taken as an indication for systematic uncertainties. If, indeed, the physical reality does not generate events in

\footnotetext{
${ }^{4}$ Such issues have been discussed in the context of a Monte Carlo generator in $[62,76]$.
} 
accordance with the functional shape of the generalized parton model, then using the model for the extraction of distributions necessarily involves systematic errors. Again, we point out that it is unclear whether the modifications we have implemented in our MC bring us closer to the physical reality. Nonetheless, the modifications are reasonable and so we believe they can give us a hint about the order of magnitude of systematic errors from the corresponding approximations in the model. One can then estimate that for calculations such as those performed in ref. [71], systematic errors in the comparison with experimental data for $b_{T}<6 \mathrm{GeV}^{-1}$ are of the order of a few percent. For the data with $b_{T}>6 \mathrm{GeV}^{-1}$, the effects of four-momentum conservation (difference between red and green points) becomes more pronounced, and a fit of data using the parton model, i.e., without manifest four-momentum conservation, therefore becomes less accurate.

\section{Conclusions}

We have presented the first studies of Bessel-weighted asymmetries using a multidimensional Monte Carlo generator based on the fully differential cross section for TMD studies using the tree level parton model [29]. Two models have been used in the simulation; a modified Gaussian and a power law tail, for the distribution and fragmentation functions. The Bessel-weighted sums of double polarization observables, in particular, provide access to transverse momentum dependencies of partonic distributions $f_{1}$ and $g_{1 L}$. Bessel-weighted asymmetries (described in [39]) have been extracted from the generated Monte Carlo events and studies of systematic uncertainties have been performed. We observe a few percent systematic offset of the Bessel-weighted asymmetry obtained from Monte Carlo extraction compared to input model calculations, which is due to the limitations imposed by the energy and momentum conservation at the given energy $/ Q^{2}$.

We find that the Bessel weighting technique provides a powerful and reliable tool to study the Fourier transform of TMDs with controlled systematics due to experimental acceptances and resolutions with different TMD models inputs. We plan to expand our studies with more advanced parton shower and fragmentation mechanisms, as well as to include nuclear modifications in our Monte Carlo and extraction procedure.

A Monte Carlo generator including spin-orbit correlations, quark-gluon interactions and correlations between the current and target fragmentation region, which is applicable in a wide range of kinematics, will be crucial for both experimental techniques and phenomenology of Fourier transformed TMDs. Moreover, evolution equations for the distributions are typically formulated directly in coordinate (Fourier) space [16-23]. Phenomenological studies can then be performed in this space, see for example [57, 58]. Thus, the study of the scale dependence of Bessel weighted asymmetries should prove important in studies of evolution of TMDs. For the above stated reasons we propose Bessel weighted asymmetries as clean observables to study the scale dependence of TMD PDFs and FFs at existing (HERMES, COMPASS, JLab) and future facilities (Electron Ion Collider, JLab $12 \mathrm{GeV}$ ). 


\section{Acknowledgments}

This work is supported by the U.S. Department of Energy under Contract No. DEAC05-06OR23177 (H.A., A.P., P.R.), No. DE-FG02-07ER41460 (L.G.), Science without borders young talent program from CAPES (contract number 150324 da CAPES), EU FP7 (HadronPhysics3, Grant Agreement number 283286) (M.A.), and the Italian Istituto Nazionale di Fisica Nucleare (M.A., H.A., E.De-S., M.M., P.R.). We thank M. Anselmino, D. Boer, S. Brodsky, U. D'Alesio, D. Hasch, A. Kotzinian, H. Matevosyan, and S. Melis for useful and stimulating discussions. We would like to thank the referee of this paper for his/her thoughtful comments that helped us to sharpen physics discussion and improve presentation of results.

\section{A Bessel weighting}

In this appendix we review the Bessel weighting framework, and the procedure to calculate the Bessel-weighted asymmetry for the longitudinally polarized beam and target, for a given set of experimental events which is expressed in eq. (2.11).

From eq. (2.4) the SIDIS cross section written in terms of the Fourier transformed TMD PDFs and FFs [39] for the leading twist unpolarized and doubly longitudinal polarized structure functions is given by

$$
\frac{d \sigma}{d x d y d \psi d z d \phi_{h} d P_{h \perp}^{2}}=K(x, y) \int \frac{d b_{T} b_{T}}{2 \pi} J_{0}\left(b_{T} P_{h \perp}\right)\left(\mathcal{F}_{U U, T}\left(b_{T}\right)+S_{\|} \lambda_{e} \sqrt{1-\varepsilon^{2}} \mathcal{F}_{L L}\left(b_{T}\right)\right)
$$

where $K(x, y)$ is given in eq. (3.3) and where $\left|\boldsymbol{P}_{h \perp}\right| \equiv P_{h \perp}$ and $\left|\boldsymbol{b}_{T}\right| \equiv b_{T}$.

Using the Bessel weighting procedure, which in this case amounts to weighting with $J_{0}$, we write the cross section $\tilde{\sigma}\left(B_{T}\right)$ in $B_{T}$ space, in terms of the structure functions ${ }^{5} \mathcal{F}_{U U, T}$ and $\mathcal{F}_{L L}$

$$
\begin{aligned}
\tilde{\sigma}\left(B_{T}\right) & =2 \pi \int d P_{h \perp} P_{h \perp} J_{0}\left(B_{T} P_{h \perp}\right) \frac{d \sigma}{d x d y d \psi d z d \phi_{h} d P_{h \perp} P_{h \perp}} \\
& =2 \pi \int d P_{h \perp} P_{h \perp} J_{0}\left(B_{T} P_{h \perp}\right) \int \frac{d b_{T} b_{T}}{2 \pi} J_{0}\left(b_{T} P_{h \perp}\right)\left(\mathcal{F}_{U U, T}+S_{\|} \lambda_{e} \sqrt{1-\varepsilon^{2}} \mathcal{F}_{L L}\right) \\
& =K(x, y)\left(\mathcal{F}_{U U, T}+S_{\|} \lambda_{e} \sqrt{1-\varepsilon^{2}} \mathcal{F}_{L L}\right),
\end{aligned}
$$

where the structure functions in $b_{T}$ space are given by the products of Fourier transformed TMDs [39],

$$
\mathcal{F}_{U U, T}=x \sum_{a} e_{a}^{2} \tilde{f}_{1}^{a}\left(x, z^{2} b_{T}^{2}\right) \tilde{D}_{1}^{a}\left(z, b_{T}^{2}\right), \mathcal{F}_{L L}=x \sum_{a} e_{a}^{2} \tilde{g}_{1 L}^{a}\left(x, z^{2} b_{T}^{2}\right) \tilde{D}_{1}^{a}\left(z, b_{T}^{2}\right) .
$$

Labeling the cross section with \pm for the double longitudinal spin combinations $S_{\|} \lambda_{e}= \pm 1$ we have

$$
\tilde{\sigma}^{ \pm}\left(b_{T}\right)=K(x, y)\left(\mathcal{F}_{U U, T} \pm \sqrt{1-\varepsilon^{2}} \mathcal{F}_{L L}\right) .
$$

\footnotetext{
${ }^{5}$ We have suppressed the dependence on the phase space variables $x, y, z$.
} 
The Bessel weighted double spin asymmetry is $b_{T}$ space is,

$$
A_{L L}^{J_{0}\left(b_{T} P_{h \perp}\right)}\left(b_{T}\right)=\frac{\tilde{\sigma}^{+}\left(b_{T}\right)-\tilde{\sigma}^{-}\left(b_{T}\right)}{\tilde{\sigma}^{+}\left(b_{T}\right)+\tilde{\sigma}^{-}\left(b_{T}\right)} \equiv \frac{\tilde{\sigma}_{L L}\left(b_{T}\right)}{\tilde{\sigma}_{U U}\left(b_{T}\right)}=\sqrt{1-\varepsilon^{2}} \frac{\sum_{a} e_{a}^{2} \tilde{g}_{1 L}^{a}\left(x, z^{2} b_{T}^{2}\right) \tilde{D}_{1}^{a}\left(z, b_{T}^{2}\right)}{\sum_{a} e_{a}^{2} \tilde{f}_{1}^{a}\left(x, z^{2} b_{T}^{2}\right) \tilde{D}_{1}^{a}\left(z, b_{T}^{2}\right)} .
$$

Now we derive the formula to extract Bessel-weighted asymmetries by means of an event by event weighting in $\boldsymbol{P}_{h \perp}$, while binning in $x, y$, and $z$. First we express the unpolarized and doubly polarized helicity structure functions in $B_{T}$ space as

$$
\begin{aligned}
\mathcal{F}_{U U, T} & =\frac{1}{K(x, y)} \int d P_{h \perp} P_{h \perp} J_{0}\left(b_{T} P_{h \perp}\right)\left(\frac{d \sigma^{+}}{d \Phi}+\frac{d \sigma^{-}}{d \Phi}\right) \\
\mathcal{F}_{L L} & =\frac{1}{K(x, y) \sqrt{1-\varepsilon^{2}}} \int d P_{h \perp} P_{h \perp} J_{0}\left(b_{T} P_{h \perp}\right)\left(\frac{d \sigma^{+}}{d \Phi}-\frac{d \sigma^{-}}{d \Phi}\right),
\end{aligned}
$$

using the shorthand notation for the differential phase space factor $d \Phi \equiv d x d y d \psi d z$ $d P_{h \perp} P_{h \perp}$. Re-expressing the cross sections in terms of the number of events in the differential phase space "volume", eq. (A.6) is given by,

$$
\mathcal{F}_{U U, T}=\frac{1}{K(x, y)} \int d P_{h \perp} P_{h \perp} J_{0}\left(b_{T} P_{h \perp}\right)\left(\frac{1}{\mathcal{N}_{0}^{+}} \frac{d n^{+}}{d \Phi}+\frac{1}{\mathcal{N}_{0}^{-}} \frac{d n^{-}}{d \Phi}\right)
$$

and

$$
\mathcal{F}_{L L}=\frac{1}{K(x, y) \sqrt{1-\varepsilon^{2}}} \int d P_{h \perp} P_{h \perp} J_{0}\left(b_{T} P_{h \perp}\right)\left(\frac{1}{\mathcal{N}_{0}^{+}} \frac{d n^{+}}{d \Phi}-\frac{1}{\mathcal{N}_{0}^{-}} \frac{d n^{-}}{d \Phi}\right)
$$

where $d n^{ \pm}$are the number of events in a differential phase space volume, $d \Phi$, and $\mathcal{N}_{0}^{ \pm}$is the standard normalization factor, that is the product of the number of beam and target particles with \pm polarization per unit target area. In the following we assume that the experiment has been set up such that $\mathcal{N}_{0}^{+}=\mathcal{N}_{0}^{-}$.

Now we discretize the momentum integration in eq. (A.7) and (A.8) for a fixed phase space cell in $x, y, z$ such that the corresponding differential $d x d y d z$ becomes the bin volume $\Delta x \Delta y \Delta z$. Eqs. (A.7) and (A.8) thus become

$$
\begin{aligned}
\mathcal{F}_{U U, T} & =x \sum_{a} e_{a}^{2} \tilde{f}_{1}\left(x, z^{2} b_{T}^{2}\right) \tilde{D}_{1}\left(z, b_{T}^{2}\right) \\
& =\frac{1}{2}\left\{\frac{1}{\mathcal{N}_{0}^{+}} \sum_{i \in \operatorname{bin}[x, y, z]} \frac{J_{0}\left(b_{T} P_{h \perp i}\right) \Delta n_{i}^{+}}{K(x, y)}+\frac{1}{\mathcal{N}_{0}^{-}} \sum_{i \in \operatorname{bin}[x, y, z]} \frac{J_{0}\left(b_{T} P_{h \perp i}\right) \Delta n_{i}^{-}}{K(x, y)}\right\} \frac{1}{\Delta x \Delta y \Delta z},
\end{aligned}
$$

and

$$
\begin{aligned}
\mathcal{F}_{L L} & =x \sum_{a} e_{a}^{2} \tilde{g}_{1}\left(x, b_{T}^{2}\right) \tilde{D}_{1}\left(z, b_{T}^{2}\right) \\
& =\frac{1}{2}\left\{\frac{1}{\mathcal{N}_{0}^{+}} \sum_{i \in \operatorname{bin}[x, y, z]} \frac{J_{0}\left(b_{T} P_{h \perp i}\right) \Delta n_{i}^{+}}{K(x, y) \sqrt{1-\varepsilon^{2}}}-\frac{1}{\mathcal{N}_{0}^{-}} \sum_{i \in \operatorname{bin}[x, y, z]} \frac{J_{0}\left(b_{T} P_{h \perp i}\right) \Delta n_{i}^{-}}{K(x, y) \sqrt{1-\varepsilon^{2}}}\right\} \frac{1}{\Delta x \Delta y \Delta z} .
\end{aligned}
$$


where we sum over the discrete momentum index $i$, and $\Delta n_{i}^{ \pm}$are the number of events for polarization \pm as a function of $P_{h \perp i}$.

Substituting eqs. (A.9) and (A.10) into eq. (A.4), the experimental procedure to calculate the Bessel weighted asymmetry, $A_{L L}^{J_{0}\left(b_{T} P_{h \perp}\right)}\left(b_{T}\right)$, becomes,

$$
\begin{aligned}
A_{L L}^{J_{0}\left(b_{T} P_{h \perp}\right)}\left(b_{T}\right)= & \frac{\tilde{\sigma}^{+}\left(b_{T}\right)-\tilde{\sigma}^{-}\left(b_{T}\right)}{\tilde{\sigma}^{+}\left(b_{T}\right)+\tilde{\sigma}^{-}\left(b_{T}\right)} \\
& =\frac{\sum_{j}^{N^{+}} J_{0}\left(b_{T} P_{h \perp j}^{[+]}\right)-\sum_{j}^{N^{-}} J_{0}\left(b_{T} P_{h \perp j}^{[-]}\right)}{\sum_{j}^{N^{+}} J_{0}\left(b_{T} P_{h \perp j}^{[+]}\right)+\sum_{j}^{N^{-}} J_{0}\left(b_{T} P_{h \perp j}^{[-]}\right)} \\
& \equiv \frac{\tilde{S}^{+}-\tilde{S}^{-}}{\tilde{S}^{+}+\tilde{S}^{-}}
\end{aligned}
$$

where $j$ are indices for the sums on events and $N^{ \pm}$are the number of events, for positive/negative products of lepton and nucleon helicities and at given $x, y$ and $z$, and where $\tilde{S}^{ \pm}$indicate the sum over events for \pm helicities.

\section{B Error calculations}

In this section we derive a formula for the standard deviation of the experimentally measured asymmetry eqs. (2.11), (A.11). First, we need to address sums of the form

$$
\tilde{S}=\sum_{j=1}^{N} J_{0}\left(b_{T} P_{h \perp j}\right) .
$$

The number of events, $N$, can be regarded as a realization of a discrete random variable $M$ with a Poisson distribution. Our best guess for its expectation value $\mathrm{E}[M]$ is $N$. The momenta $P_{h \perp 1}, P_{h \perp 2}, \ldots$ are samples independently drawn from an unknown, continuous distribution. Thus the Bessel weights $J_{0}\left(b_{T} P_{h \perp 1}\right), J_{0}\left(b_{T} P_{h \perp 2}\right), \ldots$ are realizations of independent, identically distributed random variables $W_{1}, W_{2}, \ldots$ The entire sum $\tilde{S}$ is thus the realization of a random variable

$$
Y \equiv \sum_{j=1}^{M} W_{j}
$$

This has the form of a compound Poisson distribution, see, e.g., refs. [74, 75]. The variance of $Y$ is known to be

$$
\operatorname{Var}[Y]=\mathrm{E}[M] \mathrm{E}\left[W^{2}\right]=\mathrm{E}\left[\sum_{j=1}^{M} W_{j}^{2}\right]
$$

So an estimate of the variance can simply be obtained from the recorded events by computing

$$
\operatorname{Var}[Y] \approx(\Delta \tilde{S})^{2} \equiv \sum_{j=1}^{N} J_{0}\left(b_{T} P_{h \perp j}\right)^{2}
$$


Now we use the fact that the events for helicity products + and - are independent. Therefore, the asymmetry $A_{L L}^{J_{0}\left(b_{T} \boldsymbol{P}_{h \perp}\right)}\left(b_{T}\right)$ given by eqs. (2.11), (A.11) receives two independent contributions to its uncertainty,

$$
\Delta \tilde{S}^{+}=\sqrt{\sum_{j=1}^{N^{+}} J_{0}\left(b_{T} P_{h \perp j}^{[+]}\right)^{2}}, \quad \Delta \tilde{S}^{-}=\sqrt{\sum_{j=1}^{N^{-}} J_{0}\left(b_{T} P_{h \perp j}^{[-]}\right)^{2}} .
$$

We then apply regular error propagation to obtain the standard deviation

$$
\begin{aligned}
\Delta A_{L L}^{J_{0}\left(b_{T} \boldsymbol{P}_{h \perp}\right)}\left(b_{T}\right) & =\frac{2}{\left(\tilde{S}^{+}+\tilde{S}^{-}\right)^{2}} \sqrt{\left(\tilde{S}^{-}\right)^{2}\left(\Delta \tilde{S}^{+}\right)^{2}+\left(\tilde{S}^{+}\right)^{2}\left(\Delta \tilde{S}^{-}\right)^{2}} \\
& =\frac{1-\left(A_{L L}^{J_{0}\left(b_{T} \boldsymbol{P}_{h \perp}\right)}\left(b_{T}\right)\right)^{2}}{2} \sqrt{\left(\frac{\Delta \tilde{S}^{+}}{\tilde{S}^{+}}\right)^{2}+\left(\frac{\Delta \tilde{S}^{-}}{\tilde{S}^{-}}\right)^{2}}
\end{aligned}
$$

\section{Bootstrap technique for weighted Poisson events}

Consider a fit parameter $o$ from a fit to the asymmetries $A_{L L}^{J_{0}\left(b_{T} \boldsymbol{P}_{h \perp}\right)}\left(b_{T}\right)$ extracted for an array of values $b_{T}$. The fit parameter $o$ is just an example of an observable that is calculated from a set of intermediate results with strongly correlated statistical fluctuations. To perform an error analysis for $o$, we may turn to resampling techniques.

Imagine we could repeat the entire experiment $K$ times, where $K$ is a large number, say 1000 or 10000 . We could calculate the observable for all $K$ experiments, resulting in values $o^{(1)}, o^{(2)}, \ldots, o^{(K)}$ and then compute the sample variance according to

$$
(\Delta o)^{2}=\frac{1}{K-1} \sum_{k=1}^{K}\left(\left(o^{(k)}\right)^{2}-\bar{o}^{2}\right)
$$

where $\bar{o} \equiv K^{-1} \sum_{k=1}^{K} o^{(k)}$. This straightforward procedure gives us an estimate $\Delta o$ for the error of $o$ in the original experiment. Bootstrap resampling [77, 78] is a trick that allows us to do just that without repeating the experiment in reality. New data sets are produced from the original data using a random process that in some sense mimicks an actual repetition of the experiment.

In standard bootstrap resampling, the resampled data sets are all of the same size as the original data set. For the problem at hand, however, the sample numbers $N^{+}$and $N^{-}$ are realizations of random variables as well. An obvious adaption of the usual resampling strategy is thus to vary the size of the generated data sets randomly, leading to the following algorithm:

- For $k$ from 1 to $K$

- For both helicity products \pm

* Choose a random integer $N^{ \pm(k)}$ according to a Poisson distribution with expectation value $N^{ \pm}$. 
* Choose $N^{ \pm(k)}$ random integers $j_{1}^{(k)}, \ldots, j_{N^{ \pm(k)}}^{(k)}$ from a uniform distribution in the range $1 \ldots N^{ \pm}$(random sampling with replacement).

* Calculate $\tilde{S}^{ \pm(k)}=\sum_{l=1}^{N^{ \pm(k)}} J_{0}\left(b_{T} P_{h \perp j_{l}^{(k)}}^{[ \pm]}\right)$for any desired value of $b_{T}$.

- Calculate the asymmetries $A_{L L}^{J_{0}\left(b_{T} \boldsymbol{P}_{h \perp}\right),(k)}\left(b_{T}\right)=\left(\tilde{S}^{+(k)}-\tilde{S}^{-(k)}\right) /\left(\tilde{S}^{+(k)}+\tilde{S}^{-(k)}\right)$ for any desired value of $b_{T}$.

- Calculate all other observables $o^{(k)}$ of interest using the asymmetries obtained in the previous step for data set $k$. (This may involve fits to the $b_{T}$-dependence of the asymmetries.)

- Determine error estimates for all observables o using eq. (C.1) or similar statistical means.

By performing the error analysis "empirically" for each observable $o$ in the very last step, correlations among the observables are taken into account correctly automatically.

Consider again the case that $o$ is obtained from a fit to the $b_{T}$-dependence. Depending on the details of the fit procedure (e.g., weights or covariance matrix) the size of statistical fluctuations of $o$ may vary. However, as long as the resampled data is statistically representative, the value $\Delta o$ obtained from the bootstrap method will provide a good estimate of the expected fluctuations, appropriate for the chosen fit procedure.

The algorithm above is mathematically equivalent to the bootstrap method proposed in ref. [75], even though the algorithm described in that reference looks different. According to ref. [75], the instructions in the innermost loop of the above algorithm would instead read

- Choose $N^{ \pm}$random integers $n_{1}^{ \pm(k)}, \ldots, n_{N^{ \pm}}^{ \pm(k)}$ from a Poisson distribution with expectation value 1 .

- Calculate $\tilde{S}^{ \pm(k)}=\sum_{j=1}^{N^{ \pm}} n_{j}^{ \pm(k)} J_{0}\left(b_{T} P_{h \perp j}^{[ \pm]}\right)$for any desired value of $b_{T}$.

To proove equivalence with the algorithm further above, let $\operatorname{Poi}_{\lambda}(n) \equiv \lambda^{n} e^{-\lambda} / n$ ! denote the probability to obtain $n$ from a Poisson distribution with expectation value $\lambda$. The probability to obtain integers $n_{1}^{ \pm(k)}, \ldots, n_{N^{ \pm}}^{ \pm(k)}$ in the latter algorithm is

$$
\begin{aligned}
\operatorname{Poi}_{1}\left(n_{1}\right) \cdots \operatorname{Poi}_{1}\left(n_{N}\right) & =\frac{e^{-N}}{n_{1} ! \cdots n_{N} !}=\operatorname{Poi}_{N}(T) \frac{T !}{N^{T} n_{1} ! \cdots n_{N} !} \\
& =\operatorname{Poi}_{N}(T) T ! \prod_{j=1}^{N} \frac{(1 / N)^{n_{j}}}{n_{j} !} \\
& =\operatorname{Poi}_{N}(T) \operatorname{Multi}_{T, N}\left(n_{1}, \ldots, n_{N}\right)
\end{aligned}
$$

where we have ommitted the superscripts ${ }^{ \pm}$and ${ }^{(k)}$ for better readability, and where $T=$ $\sum_{j=1}^{N} n_{j} . \operatorname{Multi}_{T, N}\left(n_{1}, \ldots, n_{N}\right)$ is the probability to obtain the vector $\left(n_{1}, \ldots, n_{N}\right)$ from a multinomial distribution with $T$ trials and equal probabilities $1 / N$ for all $N$ categories. Now if we identify $T=N^{ \pm(k)}$, it becomes evident that the scheme of ref. [75] is indeed equivalent to first determining the number of terms $N^{ \pm(k)}$ in the sum from a Poisson distribution, and then drawing momenta $P_{h \perp j}^{[ \pm]}$randomly (with replacement) from the experimentally determined data set. 
Open Access. This article is distributed under the terms of the Creative Commons Attribution License (CC-BY 4.0), which permits any use, distribution and reproduction in any medium, provided the original author(s) and source are credited.

\section{References}

[1] J. Dudek et al., Physics Opportunities with the 12 GeV Upgrade at Jefferson Lab, Eur. Phys. J. A 48 (2012) 187 [arXiv: 1208.1244] [INSPIRE].

[2] COMPASS collaboration, F. Gautheron et al., COMPASS-II Proposal, CERN-SPSC-2010-014 (2010).

[3] H. Gao et al., Transverse Spin Structure of the Nucleon through Target Single Spin Asymmetry in Semi-Inclusive Deep-Inelastic $\left(e, e^{\prime} \pi^{ \pm}\right)$Reaction at Jefferson Lab, Eur. Phys. J. Plus 126 (2011) 2 [arXiv:1009.3803] [INSPIRE].

[4] D. Boer et al., Gluons and the quark sea at high energies: Distributions, polarization, tomography, arXiv:1108.1713 [INSPIRE].

[5] A. Accardi et al., Electron Ion Collider: The Next QCD Frontier - Understanding the glue that binds us all, arXiv:1212.1701 [INSPIRE].

[6] HERMES collaboration, A. Airapetian et al., Single-spin asymmetries in semi-inclusive deep-inelastic scattering on a transversely polarized hydrogen target, Phys. Rev. Lett. 94 (2005) 012002 [hep-ex/0408013] [INSPIRE].

[7] COMPASS collaboration, V.Y. Alexakhin et al., First measurement of the transverse spin asymmetries of the deuteron in semi-inclusive deep inelastic scattering, Phys. Rev. Lett. 94 (2005) 202002 [hep-ex/0503002] [INSPIRE].

[8] COMPASS collaboration, E.S. Ageev et al., A New measurement of the Collins and Sivers asymmetries on a transversely polarised deuteron target, Nucl. Phys. B 765 (2007) 31 [hep-ex/0610068] [INSPIRE].

[9] HERMES collaboration, A. Airapetian et al., Effects of transversity in deep-inelastic scattering by polarized protons, Phys. Lett. B 693 (2010) 11 [arXiv:1006.4221] [INSPIRE].

[10] COMPASS collaboration, M.G. Alekseev et al., Measurement of the Collins and Sivers asymmetries on transversely polarised protons, Phys. Lett. B 692 (2010) 240 [arXiv: 1005.5609] [INSPIRE].

[11] COMPASS collaboration, M.G. Alekseev et al., Azimuthal asymmetries of charged hadrons produced by high-energy muons scattered off longitudinally polarised deuterons, Eur. Phys. J. C 70 (2010) 39 [arXiv: 1007.1562] [INSPIRE].

[12] COMPASS collaboration, C. Adolph et al., Transverse spin effects in hadron-pair production from semi-inclusive deep inelastic scattering, Phys. Lett. B 713 (2012) 10 [arXiv: 1202.6150] [INSPIRE].

[13] H. Mkrtchyan et al., Transverse momentum dependence of semi-inclusive pion production, Phys. Lett. B 665 (2008) 20 [arXiv:0709.3020] [INSPIRE].

[14] CLAS collaboration, H. Avakian et al., Measurement of Single and Double Spin Asymmetries in Deep Inelastic Pion Electroproduction with a Longitudinally Polarized Target, Phys. Rev. Lett. 105 (2010) 262002 [arXiv:1003.4549] [INSPIRE]. 
[15] Jefferson Lab Hall A collaboration, X. Qian et al., Single Spin Asymmetries in Charged Pion Production from Semi-Inclusive Deep Inelastic Scattering on a Transversely Polarized ${ }^{3}$ He Target, Phys. Rev. Lett. 107 (2011) 072003 [arXiv:1106.0363] [INSPIRE].

[16] J.C. Collins and D.E. Soper, Back-To-Back Jets in QCD, Nucl. Phys. B 193 (1981) 381 [Erratum ibid. B 213 (1983) 545] [INSPIRE].

[17] J.C. Collins and D.E. Soper, Parton Distribution and Decay Functions, Nucl. Phys. B 194 (1982) 445 [INSPIRE].

[18] J.C. Collins, D.E. Soper and G.F. Sterman, Transverse Momentum Distribution in Drell-Yan Pair and $W$ and $Z$ Boson Production, Nucl. Phys. B 250 (1985) 199 [INSPIRE].

[19] X.-d. Ji, J.-p. Ma and F. Yuan, QCD factorization for semi-inclusive deep-inelastic scattering at low transverse momentum, Phys. Rev. D 71 (2005) 034005 [hep-ph/0404183] [INSPIRE].

[20] J.C. Collins and A. Metz, Universality of soft and collinear factors in hard-scattering factorization, Phys. Rev. Lett. 93 (2004) 252001 [hep-ph/0408249] [INSPIRE].

[21] A. Bacchetta, D. Boer, M. Diehl and P.J. Mulders, Matches and mismatches in the descriptions of semi-inclusive processes at low and high transverse momentum, JHEP 08 (2008) 023 [arXiv:0803.0227] [INSPIRE].

[22] S.M. Aybat and T.C. Rogers, TMD Parton Distribution and Fragmentation Functions with QCD Evolution, Phys. Rev. D 83 (2011) 114042 [arXiv:1101.5057] [INSPIRE].

[23] J. Collins, Foundations of perturbative QCD, Cambridge University Press, Cambridge U.K. (2011).

[24] A. Kotzinian, New quark distributions and semiinclusive electroproduction on the polarized nucleons, Nucl. Phys. B 441 (1995) 234 [hep-ph/9412283] [inSPIRE].

[25] P.J. Mulders and R.D. Tangerman, The Complete tree level result up to order $1 / Q$ for polarized deep inelastic leptoproduction, Nucl. Phys. B 461 (1996) 197 [Erratum ibid. B 484 (1997) 538] [hep-ph/9510301] [INSPIRE].

[26] J. Levelt and P.J. Mulders, Time reversal odd fragmentation functions in semiinclusive scattering of polarized leptons from unpolarized hadrons, Phys. Lett. B 338 (1994) 357 [hep-ph/9408257] [INSPIRE].

[27] A. Bacchetta et al., Semi-inclusive deep inelastic scattering at small transverse momentum, JHEP 02 (2007) 093 [hep-ph/0611265] [INSPIRE].

[28] D. de Florian, R. Sassot and M. Stratmann, Global analysis of fragmentation functions for pions and kaons and their uncertainties, Phys. Rev. D 75 (2007) 114010 [hep-ph/0703242] [INSPIRE].

[29] M. Anselmino et al., The Role of Cahn and sivers effects in deep inelastic scattering, Phys. Rev. D 71 (2005) 074006 [hep-ph/0501196] [INSPIRE].

[30] D. Amrath, A. Bacchetta and A. Metz, Reviewing model calculations of the Collins fragmentation function, Phys. Rev. D 71 (2005) 114018 [hep-ph/0504124] [INSPIRE].

[31] A. Bacchetta, L.P. Gamberg, G.R. Goldstein and A. Mukherjee, Collins fragmentation function for pions and kaons in a spectator model, Phys. Lett. B 659 (2008) 234 [arXiv: 0707.3372] [INSPIRE].

[32] H.H. Matevosyan, A.W. Thomas and W. Bentz, Calculating Kaon Fragmentation Functions from NJL-Jet Model, Phys. Rev. D 83 (2011) 074003 [arXiv:1011.1052] [INSPIRE]. 
[33] M. Hirai, S. Kumano, T.-H. Nagai and K. Sudoh, Determination of fragmentation functions and their uncertainties, Phys. Rev. D 75 (2007) 094009 [hep-ph/0702250] [INSPIRE].

[34] M. Anselmino et al., Sivers Effect for Pion and Kaon Production in Semi-Inclusive Deep Inelastic Scattering, Eur. Phys. J. A 39 (2009) 89 [arXiv:0805.2677] [InSPIRE].

[35] H.H. Matevosyan, A.W. Thomas and W. Bentz, Collins Fragmentation Function within NJL-jet Model, Phys. Rev. D 86 (2012) 034025 [arXiv:1205.5813] [INSPIRE].

[36] H.H. Matevosyan, W. Bentz, I.C. Cloet and A.W. Thomas, Transverse Momentum Dependent Fragmentation and Quark Distribution Functions from the NJL-jet Model, Phys. Rev. D 85 (2012) 014021 [arXiv:1111.1740] [INSPIRE].

[37] A. Casey, I.C. Cloet, H.H. Matevosyan and A.W. Thomas, Dihadron Fragmentation Functions from the NJL-jet model and their QCD Evolution, Phys. Rev. D 86 (2012) 114018 [arXiv: 1207.4267] [INSPIRE].

[38] A. Signori, A. Bacchetta, M. Radici and G. Schnell, Investigations into the flavor dependence of partonic transverse momentum, JHEP 11 (2013) 194 [arXiv:1309.3507] [INSPIRE].

[39] D. Boer, L. Gamberg, B. Musch and A. Prokudin, Bessel-Weighted Asymmetries in Semi Inclusive Deep Inelastic Scattering, JHEP 10 (2011) 021 [arXiv:1107.5294] [INSPIRE].

[40] R.N. Cahn, Azimuthal Dependence in Leptoproduction: A Simple Parton Model Calculation, Phys. Lett. B 78 (1978) 269 [INSPIRE].

[41] R.N. Cahn, Critique of Parton Model Calculations of Azimuthal Dependence in Leptoproduction, Phys. Rev. D 40 (1989) 3107 [INSPIRE].

[42] M. Gourdin, Semiinclusive reactions induced by leptons, Nucl. Phys. B 49 (1972) 501 [INSPIRE].

[43] D. Boer and P.J. Mulders, Time reversal odd distribution functions in leptoproduction, Phys. Rev. D 57 (1998) 5780 [hep-ph/9711485] [INSPIRE].

[44] M. Diehl and S. Sapeta, On the analysis of lepton scattering on longitudinally or transversely polarized protons, Eur. Phys. J. C 41 (2005) 515 [hep-ph/0503023] [INSPIRE].

[45] A. Bacchetta, U. D'Alesio, M. Diehl and C.A. Miller, Single-spin asymmetries: The Trento conventions, Phys. Rev. D 70 (2004) 117504 [hep-ph/0410050] [InSPIRE].

[46] COMPASS collaboration, C. Adolph et al., Hadron Transverse Momentum Distributions in Muon Deep Inelastic Scattering at $160 \mathrm{GeV} / \mathrm{c}$, Eur. Phys. J. C 73 (2013) 2531 [arXiv: 1305.7317] [INSPIRE].

[47] HERMES collaboration, A. Airapetian et al., Multiplicities of charged pions and kaons from semi-inclusive deep-inelastic scattering by the proton and the deuteron, Phys. Rev. D 87 (2013) 074029 [arXiv: 1212 .5407] [INSPIRE].

[48] M. Anselmino, M. Boglione, J.O. Gonzalez Hernandez, S. Melis and A. Prokudin, Unpolarised Transverse Momentum Dependent Distribution and Fragmentation Functions from SIDIS Multiplicities, JHEP 04 (2014) 005 [arXiv: 1312.6261] [INSPIRE].

[49] Z. Lu and B.-Q. Ma, Sivers function in light-cone quark model and azimuthal spin asymmetries in pion electroproduction, Nucl. Phys. A 741 (2004) 200 [hep-ph/0406171] [INSPIRE]. 
[50] M. Anselmino, A. Efremov, A. Kotzinian and B. Parsamyan, Transverse momentum dependence of the quark helicity distributions and the Cahn effect in double-spin asymmetry A(LL) in Semi Inclusive DIS, Phys. Rev. D 74 (2006) 074015 [hep-ph/0608048] [INSPIRE].

[51] B. Pasquini, S. Cazzaniga and S. Boffi, Transverse momentum dependent parton distributions in a light-cone quark model, Phys. Rev. D 78 (2008) 034025 [arXiv:0806.2298] [InSPIRE].

[52] C. Bourrely, F. Buccella and J. Soffer, Semiinclusive DIS cross sections and spin asymmetries in the quantum statistical parton distributions approach, Phys. Rev. D 83 (2011) 074008 [arXiv: 1008.5322] [INSPIRE].

[53] P. Hagler, B.U. Musch, J.W. Negele and A. Schafer, Intrinsic quark transverse momentum in the nucleon from lattice QCD, Europhys. Lett. 88 (2009) 61001 [arXiv:0908.1283] [INSPIRE].

[54] B.U. Musch, P. Hagler, J.W. Negele and A. Schafer, Exploring quark transverse momentum distributions with lattice QCD, Phys. Rev. D 83 (2011) 094507 [arXiv: 1011.1213] [INSPIRE].

[55] P. Schweitzer, M. Strikman and C. Weiss, Intrinsic transverse momentum and parton correlations from dynamical chiral symmetry breaking, JHEP 01 (2013) 163 [arXiv:1210.1267] [INSPIRE].

[56] A.M. Kotzinian and P.J. Mulders, Probing transverse quark polarization via azimuthal asymmetries in leptoproduction, Phys. Lett. B 406 (1997) 373 [hep-ph/9701330] [INSPIRE].

[57] A.V. Konychev and P.M. Nadolsky, Universality of the Collins-Soper-Sterman nonperturbative function in gauge boson production, Phys. Lett. B 633 (2006) 710 [hep-ph/0506225] [INSPIRE].

[58] C.A. Aidala, B. Field, L.P. Gamberg and T.C. Rogers, Limits on transverse momentum dependent evolution from semi-inclusive deep inelastic scattering at moderate $Q$, Phys. Rev. D 89 (2014) 094002 [arXiv:1401.2654] [INSPIRE].

[59] P. Schweitzer, T. Teckentrup and A. Metz, Intrinsic transverse parton momenta in deeply inelastic reactions, Phys. Rev. D 81 (2010) 094019 [arXiv: 1003.2190] [InSPIRE].

[60] M. Boglione, S. Melis and A. Prokudin, Partonic Transverse Motion in Unpolarized Semi-Inclusive Deep Inelastic Scattering Processes, Phys. Rev. D 84 (2011) 034033 [arXiv:1106.6177] [INSPIRE].

[61] J.C. Collins, T.C. Rogers and A.M. Stasto, Fully unintegrated parton correlation functions and factorization in lowest-order hard scattering, Phys. Rev. D 77 (2008) 085009 [arXiv: 0708.2833] [INSPIRE].

[62] J. Collins and H. Jung, Need for fully unintegrated parton densities, hep-ph/0508280 [INSPIRE].

[63] S. Jadach, Foam: A general-purpose cellular Monte Carlo event generator, Comput. Phys. Commun. 152 (2003) 55.

[64] S.J. Brodsky, Light-Front Holography and the Light-Front Schrödinger Equation, University of Warsaw, Warsaw Poland, 3-6 July (2012).

[65] G.F. de Teramond and S.J. Brodsky, Light-Front Holography: A First Approximation to QCD, Phys. Rev. Lett. 102 (2009) 081601 [arXiv:0809.4899] [INSPIRE].

[66] L.P. Gamberg, G.R. Goldstein and M. Schlegel, Transverse Quark Spin Effects and the Flavor Dependence of the Boer-Mulders Function, Phys. Rev. D 77 (2008) 094016 [arXiv:0708.0324] [INSPIRE]. 
[67] H.H. Matevosyan, A. Kotzinian and A.W. Thomas, Studies of Azimuthal Modulations in Two Hadron Fragmentation of a Transversely Polarised Quark, Phys. Lett. B 731 (2014) 208 [arXiv: 1312.4556] [INSPIRE].

[68] B. Pasquini and S. Boffi, Electroweak structure of the nucleon, meson cloud and light-cone wavefunctions, Phys. Rev. D 76 (2007) 074011 [arXiv:0707.2897] [INSPIRE].

[69] B. Pasquini, S. Boffi, A.V. Efremov and P. Schweitzer, Transverse momentum dependent parton distributions and azimuthal asymmetries in light-cone quark models, arXiv:0912.1761 [INSPIRE].

[70] HERMES collaboration, A. Airapetian et al., Azimuthal distributions of charged hadrons, pions and kaons produced in deep-inelastic scattering off unpolarized protons and deuterons, Phys. Rev. D 87 (2013) 012010 [arXiv:1204.4161] [INSPIRE].

[71] Z. Lu and B.-Q. Ma, Quark helicity distributions in transverse momentum space and transverse coordinate space, Phys. Rev. D 87 (2013) 034037 [arXiv:1212.6864] [InSPIRE].

[72] CLAS collaboration, B.A. Mecking et al., The CEBAF Large Acceptance Spectrometer (CLAS), Nucl. Instrum. Meth. A 503 (2003) 513 [INSPIRE].

[73] E.-C. Aschenauer et al., The RHIC SPIN Program: Achievements and Future Opportunities, arXiv: 1304.0079 [INSPIRE].

[74] R. Adelson, Compound poisson distributions, Oper. Res. Q. 17 (1966) 73.

[75] G. Bohm and G. Zech, Statistics of weighted Poisson events and its applications, Nucl. Instrum. Meth. A 748 (2014) 1 [arXiv:1309.1287] [INSPIRE].

[76] J.C. Collins and X. Zu, Initial state parton showers beyond leading order, JHEP 03 (2005) 059 [hep-ph/0411332] [INSPIRE].

[77] B. Efron, Bootstrap methods: another look at the jackknife, Ann. Stat. 7 (1979) 1.

[78] B. Efron, The jackknife, the bootstrap, and other resampling plans, Society for Industrial and Applied Mathematics, Philadelphia U.S.A. (1982). 